\title{
稀土配合物/聚合物白光材料的研究进展
}

\author{
王 军*, $a$ 李小成 ${ }^{a}$ 初红涛 $b$ 何进军 ${ }^{a}$ 陈志娇 ${ }^{a}$

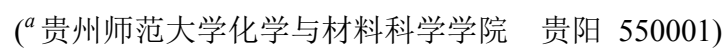 \\ $\left({ }^{b}\right.$ 齐齐哈尔大学化学与化学工程学院 黑龙江齐齐哈尔 161006)
}

\begin{abstract}
摘要 由于良好的发光性能和显色性能, 光色可调的稀土配合物/聚合物白光材料近年来受到高度关注. 根据白光材料 聚集状态以及配位聚集体中稀土离子种类的不同，重点综述 2014 2018 年来稀土配合物/聚合物白光材料的研究进展， 展望了稀土配合物/聚合物白光材料的发展趋势.
\end{abstract}

关键词 稀土配合物/聚合物; 白光发射; 光色可调; 配位组装; 光学性能

\section{Research Progress in the White Light-Emitting Lanthanide-Based Complex/Coordination Polymer Materials}

\author{
Wang, Jun ${ }^{*, a} \quad$ Li, Xiaocheng $^{a} \quad$ Chu, Hongtao ${ }^{b} \quad$ He, Jinjun $^{a} \quad$ Chen, Zhijiao $^{a}$ \\ ( ${ }^{a}$ College of Chemistry and Material Science, Guizhou Normal University, Guiyang 550001) \\ ( ${ }^{b}$ College of Chemistry and Chemical Engineering, Qiqihaer University, Qiqihaer, Heilongjiang 161006)
}

\begin{abstract}
Color-tunable and white light-emitting lanthanide-based complex/coordination polymer materials have attracted increasing interest recently due to the exceptional luminescent and chromogenic properties. Recent advances from 2014 to 2018 on white-light-emitting lanthanide-based materials are classifiedly highlighted in light of different aggregate states and lanthanide species in the coordination assemblies. The future advances in this field are also predicted.

Keywords lanthanide-based complex/coordination polymer; white light-emitting; color-tunable lumilensence; coordination assembly; optical property
\end{abstract}

近年来，由于在平板显示、发光二极管、照明、通 信等领域的广泛应用, 光色可调的发光材料, 尤其是白 光材料引起学术界以及产业界的高度关注 ${ }^{[1 \sim 5]}$. 白光二 极管(WLED)作为新型的固态发光光源, 直接被认为是 照明领域的又一次革新 ${ }^{[6]}$, 但传统的含录白光光源存在 诸多的环境问题. 因此, 环境友好、高效节能的白光材 料已成为国内外相关工作者的追求目标. 目前已报道的 白光材料主要包括纳米材料 ${ }^{[7]}$ 、无机 ${ }^{[8]}$ 、有机 ${ }^{[9]}$ 以及有机 配体敏化的无机-有机杂化白光材料 ${ }^{[10]}$ 等. 其中, 有机 配体与稀土离子 $\left(\mathrm{Ln}^{3+}\right)$ 经由配位驱动组装形成的单相稀 土白光材料更是因为配体结构的多样性以及发光可预 见性、热稳定性、良好的显色性能、高的色纯度以及简 单的制备过程等优势而备受青睐 ${ }^{[5,11]}$. 另外, 稀土白光
材料较长的荧光寿命以及较大的 stokes 位移使其在光学 器件和光转化材料中具有较高的应用价值 ${ }^{[12]}$, 与物理 掺杂或其它白光材料相比, 能够通过控制光色不同的 $\mathrm{Ln}^{3+}$ 的掺杂量而使光色可调 ${ }^{[13]}$, 而且此类材料往往具有 温度、激发波长、溶剂等依赖性, 这为多刺激响应性智 能白光材料的制备提供了依据, 尤其是基于 $\mathrm{Ln}^{3+}$ 的金 属有机框架材料(LnOFs), 因为其独特的发光性能, 已 成为多色发光以及白光发射的明星材料 ${ }^{[13,14]}$.

$\mathrm{Ln}^{3+}$ 由于 $4 \mathrm{f}$ 电子层的 $\mathrm{f} \rightarrow \mathrm{f}$ 跃迁 (偶有 $\mathrm{d} \rightarrow \mathrm{f}$ 跃迁产生 宽带发射 ${ }^{[15]}$, 如 $\mathrm{Ce}^{3+}$ )产生基于金属中心的窄带发射, 但这种禁阻跃迁使其在可见光区表现出较弱的吸收(摩 尔吸光系数小于 $10 \mathrm{~L} \cdot \mathrm{mol}^{-1} \cdot \mathrm{cm}^{-1}$ ), 因而 $\mathrm{Ln}^{3+}$ 发光能力 较弱, 而有机配体因为具有良好的光吸收能力, 经由

\footnotetext{
* Corresponding author. E-mail: beyoundme@126.com

Received April 8, 2019; revised July 11, 2019; published online July 24, 2019.

Project supported by the Joint Funds of Science and Technology of Guizhou Province (No. [2015]7774), the Growth Project for Youth Science and Technology Talent of Education Department of Guizhou Province (No. [2016]141), the Science and Technology Project of Education Department of Heilongjiang Province (No. 12521607).

贵州省科技厅联合基金(No. [2015]7774)、贵州省教育厅青年科技人才成长项目(No. [2016]141)和黑龙江省教育厅科技(No. 12521607)资助项目.
} 
“天线效应” [16]能够敏化 $\mathrm{Ln}^{3+}$ 发光, 其实质是能量给(有 机配体) 受 $\left(\mathrm{Ln}^{3+}\right)$ 体对之间的能量转移. 传统的从配体单 重激发态(最低能级的 $\mathrm{S}_{1}$ ) $\rightarrow$ 三重激发态(最低能级的 $\mathrm{T}_{1}$ ) $\rightarrow \mathrm{Ln}^{3+}$ 的能量转移敏化机制(图 1)认为 ${ }^{[17,18]}$, 配体分 子通过紫外辐射后从基态 $\left(\mathrm{S}_{0}\right)$ 跃迁至激发态, 并经由内 部转换(IC)至最低能级激发态 $\left(\mathrm{S}_{1}\right)$, 由 $\mathrm{S}_{1}$ 到 $\mathrm{S}_{0}$ 的辐射跃 迁产生基于配体中心的苂光发射 ${ }^{[19]}$, 而通过非辐射系 间鼌跃(ISC)后最低能级 $T_{1}, S_{1}$ 与 $T_{1}$ 之间适当的能级差 $\left(\Delta E \approx 5000 \mathrm{~cm}^{-1}\right)$ 有利于传能速率和效率的提高 ${ }^{[5,20]}$, 由 $\mathrm{T}_{1}$ 到 $\mathrm{S}_{0}$ 的辐射跃迁产生基于配体中心的磷光. 当配体 $\mathrm{T}_{1}$ 能级与 $\mathrm{Ln}^{3+}$ 最低发射能级相匹配时敏化 $\mathrm{Ln}^{3+}$ 发光, 如配体敏化 $\mathrm{Eu}^{3+}\left[E\left({ }^{5} \mathrm{D}_{0}\right)=17500 \mathrm{~cm}^{-1}\right]$ 和 $\mathrm{Tb}^{3+}$ $\left[E\left({ }^{5} \mathrm{D}_{4}\right)=20500 \mathrm{~cm}^{-1}\right]$ 发光时, 能级差分别介于 $2500 \sim$ 3500 和 $2500 \sim 4000 \mathrm{~cm}^{-1}$ 之间, 两者间能级差太大往往 表现出基于配体中心的荧光发射, 当能级差太小(甚至 $\mathrm{T}_{1}$ 能级小于 $\mathrm{Ln}^{3+}$ 最低发射能级)则发生从 $\mathrm{Ln}^{3+}$ 到配体的 能量逆转移 ${ }^{[21]}$. 配体与 $\mathrm{Ln}^{3+}$ 间能量匹配程度以及敏化 发光路径对稀土发光材料发光效率的影响机制称为 “能 量匹配原则”。除此之外, 文献中已报道了 $\mathrm{S}_{1} \rightarrow$

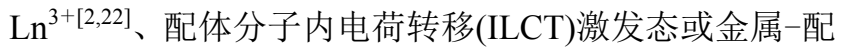
体电荷转移(MLCT)激发态 $\rightarrow \mathrm{Ln}^{3+[23,24]}$ 以及不同 $\mathrm{Ln}^{3+}$ 间 $\left(\text { 如 } \mathrm{Tb}^{3+} \rightarrow \mathrm{Eu}^{3+}\right)^{[25]}$ 的能量转移路径.

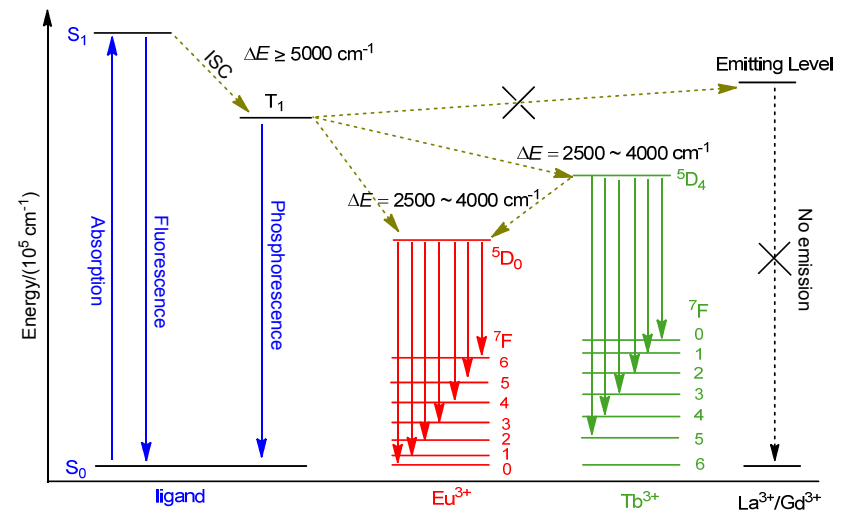

图 1 稀土 $\left(\mathrm{Eu}^{3+}, \mathrm{Tb}^{3+}, \mathrm{La}^{3+} / \mathrm{Gd}^{3+}\right)$ 配合物/聚合物能量吸收、转 移和辐射过程示意图

Figure 1 Schematic representation of the energy adsorption, migration and emission process of the lanthanide $\left(\mathrm{Eu}^{3+}, \mathrm{Tb}^{3+}\right.$, $\left.\mathrm{La}^{3+} / \mathrm{Gd}^{3+}\right)$ complexes

\section{1 稀土白光材料的调制策略及配体结构调控对 发光性能的影响}

\section{1 稀土白光材料的调制策略}

众所周知, 理想白光的色坐标 (CIE 坐标) 为 $(0.33$, 0.33 ), 相对色温(CCT)介于 $2500 \sim 6500 \mathrm{~K}$ 之间, 显色指 数 $(\mathrm{CRI})$ 大于 $80^{[26]}$, 通常由覆盖整个可见光区 $(400 \sim 700$ $\mathrm{nm}$ )的二基色(蓝光和黄光)或三基色(红、绿、蓝)复合而
成，此为白光调制的 “光色互补” 原则. 经由稀土配合 物/聚合物调制白光时，主要有以下三种调制策略: (1)对 于异核稀土配合物/聚合物白光材料, 通常由配体中心 的蓝光、 $\mathrm{Eu}^{3+}$ 的红光以及 $\mathrm{Tb}^{3+}$ 的绿光复合得到三色白 光 ${ }^{[27,28] ;}$; (2)对于异多核稀土配合物/聚合物白光材料, 由 于 $\mathrm{La}^{3+} / \mathrm{Gd}^{3+}$ 较高的发射能级很难被敏化发光, 对应的 配合物/聚合物表现出基于配体中心的蓝光发射, 当 $\mathrm{La}^{3}$ ${ }^{+} / \mathrm{Gd}^{3+}$ 配合物/聚合物进一步与 $\mathrm{Eu}^{3+} 、 \mathrm{~Tb}^{3+}$ 配位组装后, 同样能够调制得到三色白光 ${ }^{[29-33]}$. 相对而言, 多种稀土 离子参与的三色白光往往比单色或二色白光具有更高 的色纯度和显色指数 ${ }^{[10,34]}$, 但其调制成本较高; (3)对于 单/同核稀土配合物/聚合物白光材料，通常使用光色相 同或相近的非稀土金属离子 ${ }^{[35]}$ 、有机荧光团替代 $\mathrm{Ln}^{3+}$, 或直接利用配体的单体(蓝光发射)及其激基缔合物(绿 光发射) 以及稀土离子的红光(如 $\mathrm{Eu}^{3+}$ 和 $\mathrm{Sm}^{3+}$ ) 调制白 光 ${ }^{[36,37]}$, 当然也可利用蓝光配体直接与多种光色的 $\mathrm{Ln}^{3+}$ (如 $\mathrm{Sm}^{3+}$, Dy ${ }^{3+}$ 等) 调制. 相对与异/异多核白光材料而言, 单/同核稀土配合物/聚合物白光材料调制成本较低, 但 遗憾的是此类白光材料的报道相对较少 ${ }^{[2,5,38]}$. 正是因为 “光色互补与能量匹配原则”的限制, 稀土白光材料具有 普遍较低的发光效率, 光量子产率超过 $30 \%$ 的此类白光 材料鲜有报道 ${ }^{[3]}$.

\section{2 配体结构调控对发光性能的影响}

经由稀土配合物/聚合物调制白光时，首先应该考 虑敏化配体的结构对发光性能的影响. 以 “硬碱” $\mathrm{O}, \mathrm{N}$ 为配位点的芳香 $\pi$ 体系多齿配体 (提供蓝光组分)在满足 “硬酸” $\mathrm{Ln}^{3+}$ 配位数的同时，一方面增加稀土配合物/聚 合物的刚性, 有利于能量给受体对之间的能量转 移 ${ }^{[13,39]}$, 另一方面还能有效抑制溶剂参与配位, 消除因 $\mathrm{O}-\mathrm{H} / \mathrm{N}-\mathrm{H}$ 等高能键的振动而造成的非辐射衰减 ${ }^{[40]}$. 如杜少武等 ${ }^{[41]}$ 利用吡啶二甲酸构筑的异多核稀土金属 有机框架白光材料光量子产率高达 $62 \%$. 虽然, 配体刚 性的增强在一定程度上能够提高白光量子产率 ${ }^{[14]}$, 但 同时由于配体 $T_{1}$ 能级的降低而不利于能量转移 ${ }^{[42 ~ 44]}$. 为了从根本上解决 “能量匹配原则” 对发光效率的影响, 最有效的策略是配体通过结构调控突破传统的 $\mathrm{T}_{1}$ 能量 转移敏化机制, 实现配体 $S_{1}$ 敏化机制 ${ }^{[2,45]}$. 近期研究表 明, ILCT 激发态同样能够敏化 $\mathrm{Ln}^{3+}$ 的发光, 如苏成勇课 题组 ${ }^{[24]}$ 通过配体分子 “推-拉” 电子结构设计, 实现了 配体 ILCT 激发态以及 $\mathrm{T}_{1}$ 激发态协同敏化 $\mathrm{Dy}^{3+}$ 发光, 从 而调制得到光色可调的白光. 许辉课题组 ${ }^{[46]}$ 通过双齿 膦氧配体与 $\mathrm{Eu}^{3+}$ 的配位组装构筑了一维链状配位聚合 物电致红光膜材料, 由于链内有效的能量以及电荷转 移, 红光量子产率高达 $85 \%$, 进而以此为涂层得到发光 效率优异的 WLED. 
其次，应该考虑配体分子对稀土配合物/聚合物组 装体组装模式的调控. 通过配体分子中多重组装位点的 引入或将白光材料 “固定” 于凝胶或高分子膜材料的方 法, 抑制配体激发态因溶剂间频繁碰撞和振动偶合产生 的非辐射衰减 ${ }^{[47]}$. 如杨玉林等 ${ }^{[48]}$ 将吡啶-2,3-二甲酸与 $\mathrm{Eu}^{3+}, \mathrm{Gd}^{3+}$ 以及 $\mathrm{Tb}^{3+}$ 的组装体与聚甲基丙烯酸甲酯 (PMMA) 共掺杂, 得到光量子产率为 $17.6 \%$ 的白光. 另 外, 配体分子因 $\pi-\pi$ 堆积而形成的不同聚集状态对发光 性能存在截然不同的影响, 一方面要利用配体分子内/ 间电荷转移而对发光性能的改善作用. 如闰鹏飞等 ${ }^{[22]}$ 设计了 1,8-荎二酰亚胺功能化的配体, 进而利用配体的 单体(蓝光发射)及其激基缔合物(绿光发射)与 $\mathrm{Sm}^{3+}$ (红 光)配位组装得到光量子产率为 $11.9 \%$ 的白光材料. 与此 同时, 也要防止因聚集态的形成导致配体荧光猝灭以及 $\mathrm{Ln}^{3+}$ 间不必要的能量传递 ${ }^{[13,49]}$. 相应地, 文献中通过液

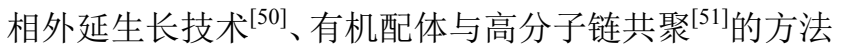
以及 LnOFs 的独特结构 ${ }^{[13]}$ 达到不同发光体空间分离的 目的. 鉴于文献[2]对白光材料的配位组装方法有详细 的介绍, 在此不再赘述.

本文在简单介绍稀土配合物/聚合物白光材料的调 制策略以及稀土发光性能的影响因素的基础上, 按其聚 集状态分类, 重点综述白光溶液、白光凝胶、白光聚合 物、白光稀土金属有机框架以及其它类基于稀土配合物 /聚合物白光材料的研究现状, 并对此类白光材料的前 景做出展望.

\section{2 白光溶液}

通过控制配体和不同 $\mathrm{Ln}^{3+}$ 间的化学计量比、溶剂的 极性以及激发波长等方法研究稀土配位组装体在溶液 状态下的发光行为, 进而调制白光. 由于溶剂对有机配 体聚集态的影响, 导致配位组装体具有光色可调的性 能.

Holten-Andersen 等 ${ }^{[52]}$ 以三联吡啶(L1)为敏化配体, 制备得到单色光发射的配合物 3L1- $\mathrm{Ln}\left(\mathrm{Ln}=\mathrm{La}^{3+}, \mathrm{Eu}^{3+}\right.$, $\left.\mathrm{Tb}^{3+}\right)$. 在 $\mathrm{H}_{2} \mathrm{O} / \mathrm{DMF}(V: V=6: 4)$ 的溶液中, $\mathrm{La}^{3+}, \mathrm{Eu}^{3+}$ 和 $\mathrm{Tb}^{3+}$ 与 $\mathbf{L 1}$ 以 $1: 1: 1: 9$ 的物质的量比混合, $365 \mathrm{~nm}$ 激发下得到白光溶液, $\mathrm{CIE}$ 坐标为 $(0.34,0.31)$. 基于 $\mathrm{Ln}-\mathrm{N}$ 的动态非共价作用, 在 $\mathrm{F}^{-}$和 $\mathrm{CN}^{-}$的刺激响应下溶 液光色从白光向蓝光转变(Eq. 2).

Gunnlaugsson 等 ${ }^{[49]}$ 设计的配体同时包含吡啶二甲 酸及三联吡啶结构单元 $(\mathbf{L 2})$, 其在甲醇溶液中具有蓝光 发射, 浓度大于 $10 \mu \mathrm{mol} \cdot \mathrm{L}^{-1}$ 时由于配体分子间的 $\pi-\pi$ 堆 积导致荧光减弱. $\mathbf{L 2}$ 与乙二胺四乙酸(EDTA) $\mathrm{Eu}^{3+} / \mathrm{Tb}^{3+}$ 配位组装形成异核稀土配合物 $\mathbf{L 2}[\mathrm{Eu}(\mathrm{EDTA})]_{x}[\mathrm{~Tb}-$ (EDTA) $]_{3-x}$ (图 2). 配合物的发光性能具有配体和 $\mathrm{Ln}^{3+}$ 浓 度以及激发波长的响应性, 当 $\mathbf{L 2}$ 浓度为 $25 \mu \mathrm{mol} \cdot \mathrm{L}^{-1}$, $\mathrm{Eu}^{3+} / \mathrm{Tb}^{3+}$ 物质的量之比为 $1: 2$, 激发波长为 $265 \sim 270$ $\mathrm{nm}$ 时, 得到色纯度较高的白光, CIE 坐标为 $(0.33,0.34)$.

间鹏飞等 ${ }^{[36]}$ 经由配体 $\mathbf{L 3}$ 和 $\mathrm{Sm}^{3+}$ 的配位组装制备了 组成为 $\mathrm{Sm}_{2}(\mathbf{L} 3)_{3}\left(\mathrm{H}_{2} \mathrm{O}\right)_{4}$ 的单核配合物, 进而利用 $\mathbf{L 3}$ 的
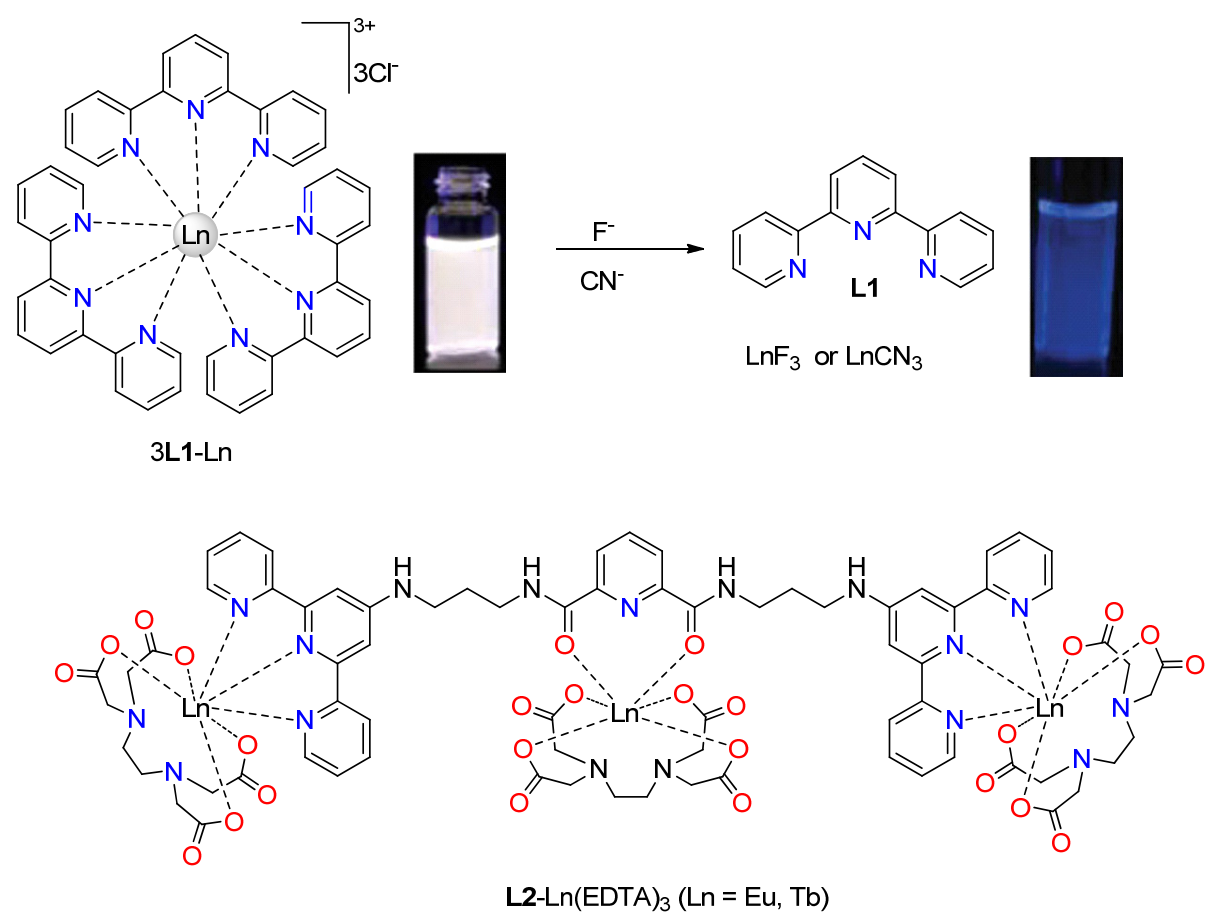

图 $2 \mathrm{L2}-\mathrm{Ln}(\mathrm{EDTA})_{3}\left(\mathrm{Ln}=\mathrm{Eu}^{3+} / \mathrm{Tb}^{3+}\right)$ 的配位组装模式

Figure 2 Coordination assembly mode of $\mathbf{L} 2-\operatorname{Ln}(\mathrm{EDTA})_{3}\left(\mathrm{Ln}=\mathrm{Eu}^{3+} / \mathrm{Tb}^{3+}\right)$ 
单体及其激基缔合物的双发射(430 和 $510 \mathrm{~nm}$ )以及 $\mathrm{Sm}^{3+}$ 的特征发射调制得到白光溶液. 浓度为 $5 \mu \mathrm{mol} \cdot \mathrm{L}^{-1}$ 的 $\mathrm{Sm}_{2}(\mathbf{L 3})_{3}\left(\mathrm{H}_{2} \mathrm{O}\right)_{4}$ 氯仿溶液在 $360 \mathrm{~nm}$ 激发下, 白光 CIE 坐标为 $(0.38,0.32)$, 量子产率为 $0.48 \%$; 而浓度为 0.15 $\mu \mathrm{mol} \cdot \mathrm{L}^{-1}$, 激发波长为 $385 \mathrm{~nm}$ 时白光 $\mathrm{CIE}$ 坐标为 $(0.34$, 0.32 ), 量子产率为 $1.43 \%$.<smiles>O=C(/C=C(\O)C(F)(F)F)c1ccc(Oc2ccc3ccccc3c2-c2c(Oc3ccc(C(=O)/C=C(\O)C(F)(F)F)cc3)ccc3ccccc23)cc1</smiles>

王苏宁课题组 ${ }^{[53]}$ 设计合成了芳基硼烷功能化的大 环多胺类配体及其稀土配合物 L4Eu， L4Tb, L5Eu 和 $\mathbf{L 5 T b}$. 室温下, $\mathbf{L 5 E u}$ 和 $\mathbf{L 5 T b}$ (物质的量之比为 $4: 6$ ) 的 甲醇溶液在 $330 \mathrm{~nm}$ 激发下, 白光 CIE 坐标为 $(0.37,0.30)$, 而 $\mathbf{L 4 E u}$ 和 $\mathbf{L 5} \mathrm{Tb}$ (物质的量之比为 $3: 7$ ) 的混合体系在甲 醇溶液中或 PMMA 掺杂下都可得到色纯度较高的白光 发射, 其 CIE 坐标为 $(0.33,0.32)$. 有趣的是白光溶液的 光色具有温度和 $\mathrm{F}^{-}$的响应性.

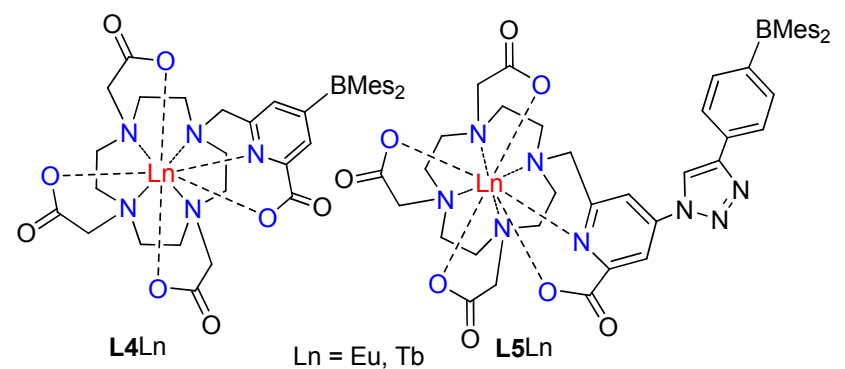

Boddula 等 ${ }^{[54]}$ 以多功能辅助配体 $\mathbf{L 6}$ (其中包含二苯 胺、三苯胺、芴以及 1,10 -邻二氮杂菲等结构单元) 以及 噻吩甲酰三氟丙酮(TTA)配体构筑了 $\mathrm{Eu}^{3+}$ 的三元配合物

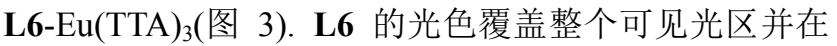
$517 \mathrm{~nm}$ 出现最大发射, 配合物的光色具有温度、溶剂和 激发波长的响应性, 其中在甲苯溶液中, $380 \mathrm{~nm}$ 激发下 得到 CIE 坐标为 $(0.34,0.33)$ 的白光, 量子产率为 $15.3 \%$, $\mathrm{CCT}$ 为 $5152 \mathrm{~K}$. 该课题组 ${ }^{[12]}$ 利用相同的策略构筑了基 于三苯胺功能化的咪唑并联吡啶(L7)以及 TTA 的三元 配合物 L7-Eu(tta) 3 (图 4), 该配合物在氯仿溶液、固态和 PMMA 掺杂的薄膜材料中得到色纯度较差的白光发射, CIE 坐标分别为 $(0.38,0.29),(0.32,0.44)$ 和(0.58, 0.30). 作者以 $\mathrm{L} 7-\mathrm{Eu}(\mathrm{tta})_{3}$ 与 PMMA(质量比为 $3: 10$ ) 的膜材料 为涂层制备了 WLED, 白光色坐标为 $(0.30,0.33)$.

Al-Rasbi 等 ${ }^{[37}$ 通过 $\mathrm{H}_{2} \mathbf{L 8}$ 和 $\mathrm{Eu}(\mathrm{HFAC})_{3} \cdot 2 \mathrm{H}_{2} \mathrm{O}$ (HFAC 为六氟乙酰丙酤)配位组装得到组成为 $\left[\mathrm{Eu}_{2}(\mathbf{L 8})_{4}-\right.$

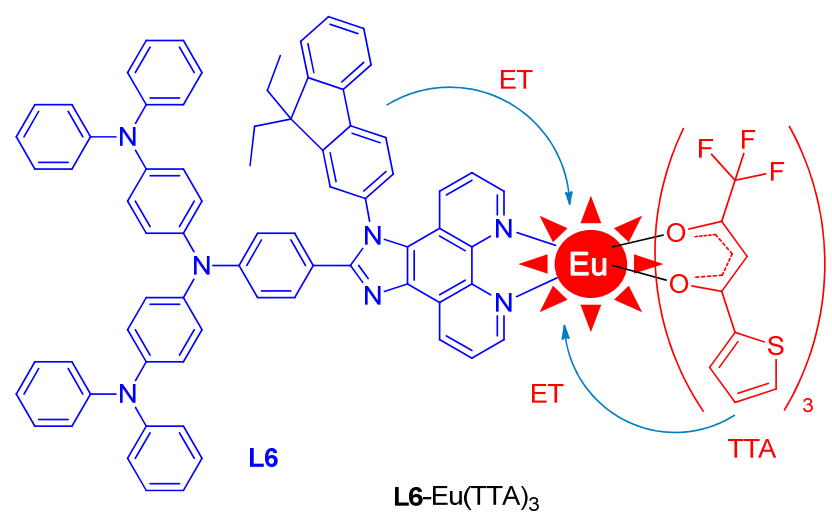

图 3 配合物 L6-Eu(TTA) $)_{3}$ 的配位组装模式

Figure 3 Coordination assembly mode of $\mathbf{L 6}$-Eu(TTA $)_{3}$

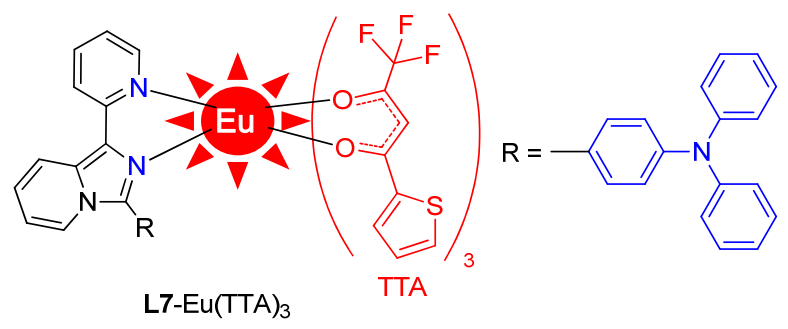

图 4 配合物 L7-Eu(TTA) 3 的配位组装模式

Figure 4 Coordination assembly mode of L7-Eu(TTA $)_{3}$

( $\mathrm{HFAC})]\left(\mathrm{O}_{2} \mathrm{CCF}_{3}\right)$ 的配合物, 其浓度为 $10 \mathrm{mmol} \cdot \mathrm{L}^{-1}$ 的 二氯甲烷溶液在 $368 \mathrm{~nm}$ 激发下, 经由配体及其激基缔 合物的双发射以及 $\mathrm{Eu}^{3+}$ 的红光复合得到白光, $\mathrm{CIE}$ 坐标 为 $(0.35,0.35)$. 配体 $\mathrm{H}_{2} \mathbf{L 9}$ 与等物质的量的 $\mathrm{Eu}^{3+}$ 和 $\mathrm{Tb}^{3+}$ 形成的异核稀土配合物 EuTbL9(HL9) 在 $340 \mathrm{~nm}$ 激发 下, 白光 CIE 坐标为 $(0.35,0.33)^{[44]}$.

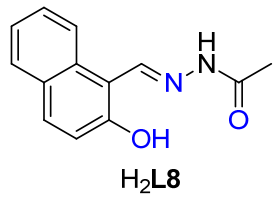<smiles>CC(=O)N/N=C/c1ccccc1O</smiles>

\section{3 聚合物白光材料}

目前文献中报道的稀土聚合物白光材料主要有两 种不同的制备方法，一是用配体功能化的高分子共聚物 与 $\mathrm{Ln}^{3+}$ 配位组装; 二是经由配体与 $\mathrm{Ln}^{3+}$ 配位聚合得到 聚合物材料，包括金属簇和 LnOFs 材料. 由于 LnOFs 篇 幅较多，因而在后文单独介绍.

吕兴强等 ${ }^{[14]}$ 以乙烯苯基联二吡啶为能量给体, $\beta$-二 酮为辅助配体，分别制备得到 $\mathrm{Eu}^{3+}$ 和 $\mathrm{Gd}^{3+}$ 的异核配合 物(分别发射红光和青色光). 两种配合物与咔唑经由自 由基聚合得到聚合物 L10-Eu-Gd(图 5). 进而通过精确 控制 $\mathrm{Eu}^{3+}$ 和 $\mathrm{Gd}^{3+}$ 浓度或改变激发波长的方法调制得到 白光, $\mathrm{CIE}$ 坐标为 $(0.33,0.33)$, 量子产率为 $18.4 \%$. 之后, 
该课题组 ${ }^{[5]}$ 将 2-吡啶基苯并咪唑与 $\mathrm{Ln}^{3+}\left(\mathrm{Eu}^{3+} / \mathrm{Tb}^{3+} /\right.$ $\mathrm{Gd}^{3+}$ )的配合物经自由基聚合反应链接在甲基丙烯酸甲 酯(MMA)上, 制备得到 1 白光聚合物材料 L11-Eu-Tb$\operatorname{Gd}$ (图 6). 保持 MMA 与稀土配合物的物质的量之比为 $200: 1$, 控制 $\mathrm{Eu}^{3+} / \mathrm{Tb}^{3+} / \mathrm{Gd}^{3+}$ 的物质的量之分别为 $1:$ $7: 4$ 和 $1: 7: 8$, 前者在 $360 \mathrm{~nm}$ 激发下白光 CIE 坐标、 CCT、CRI 以及量子产率分别为 $(0.35,0.32), 4769 \mathrm{~K}, 85$
和 9.6\%; 后者在 $345 \mathrm{~nm}$ 激发下白光对应的参数分别为 $(0.32,0.33) 、 5979 \mathrm{~K} 、 94$ 和 $17.8 \%$. 该课题组得到的白 光材料与其它类似的白光聚合物材料 ${ }^{[56]}$ 相比, 具有更 好的色纯度以及更高的发光效率.

苏成勇课题组 ${ }^{[11]}$ 首先合成了乙烯基苄基修饰的三 脚架配体分子与 $\mathrm{Ln}^{3+}$ 的配合物, 进而将配合物与 MMA 共聚得到金属共聚物 L12- $\operatorname{Ln}$ (图 7), 作者认为这种金属

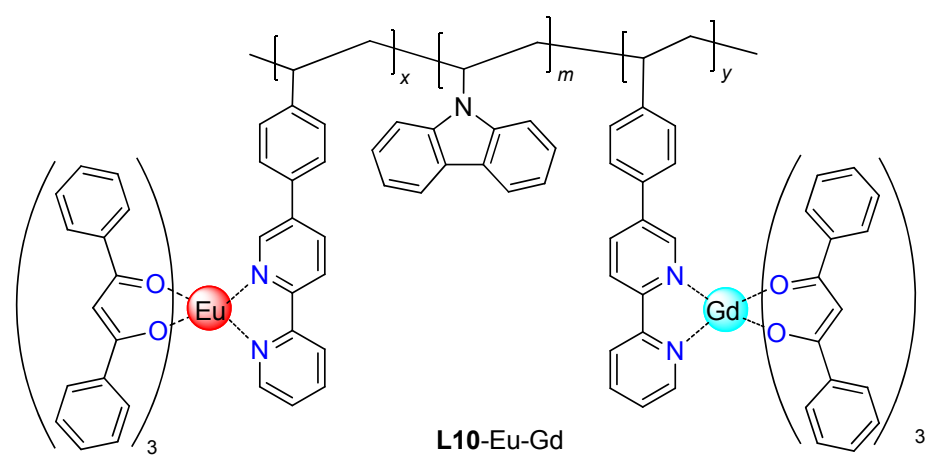

图 5 聚合物 L10-Eu-Gd 的配位组装模式

Figure 5 Coordination assembly mode of L10-Eu-Gd polymer

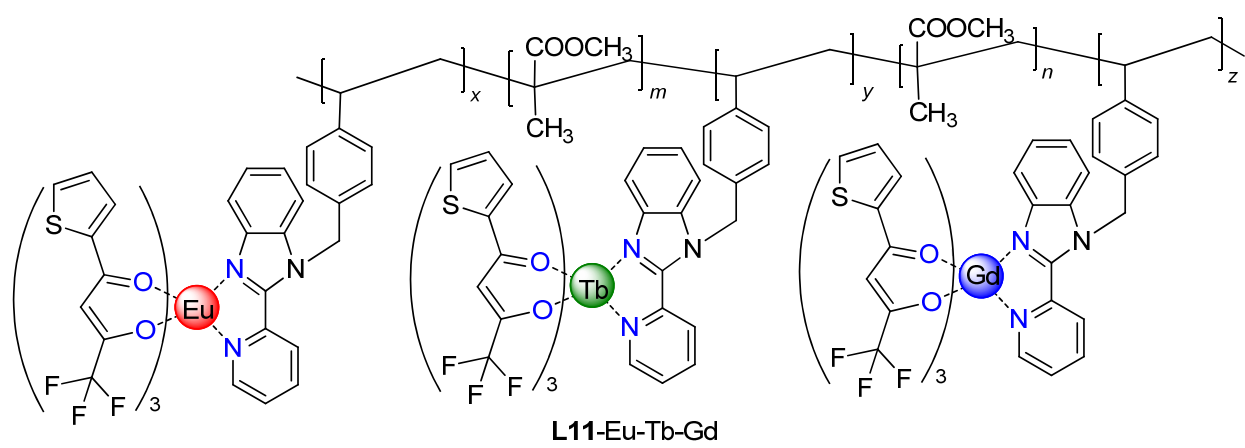

图 6 聚合物 L11-Eu-Tb-Gd 的配位组装模式

Figure 6 Coordination assembly mode of L11-Eu-Tb-Gd polymer
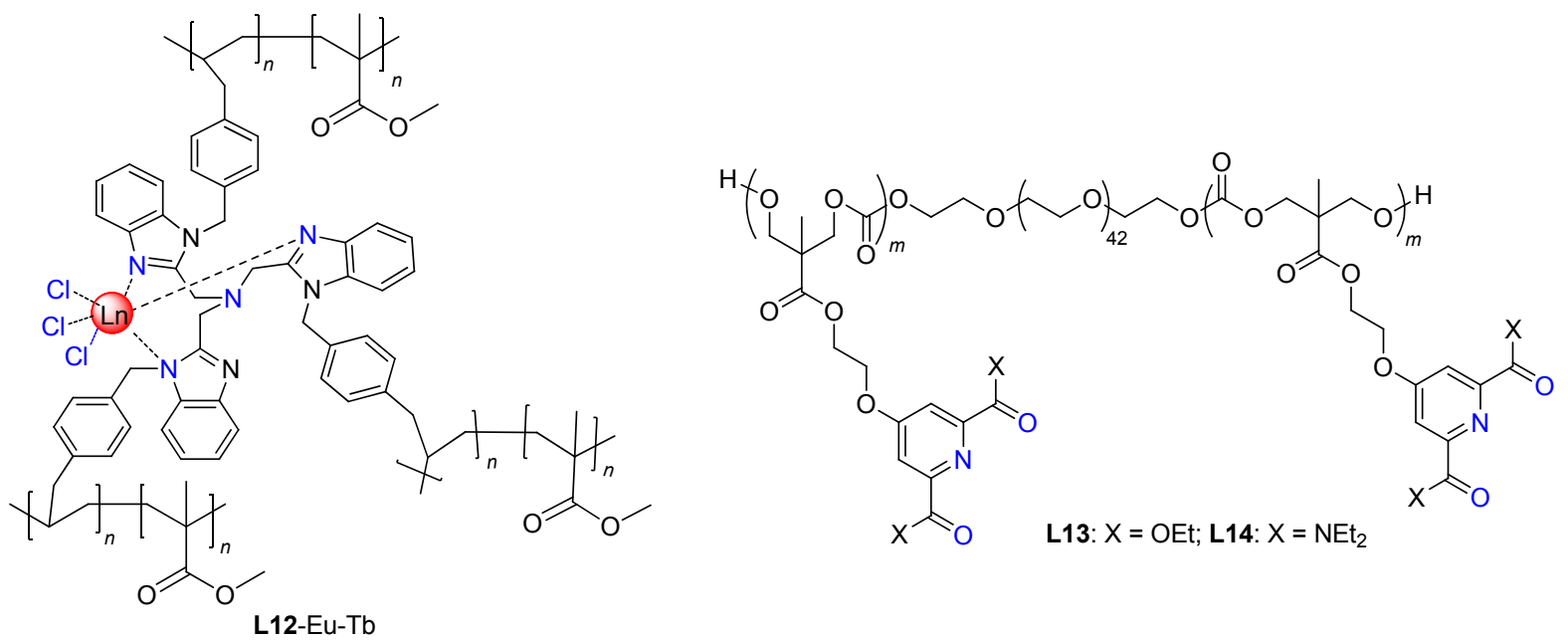

图 7 聚合物 L12-Eu-Tb 配位组装模式和蓝光配体 L13 及 $\mathbf{L 1 4}$ 的结构示意图

Figure 7 Coordination assembly mode of L12-Eu-Tb polymer, and structures of blue light ligands L13 and L14 
共聚物的生成有利于配体 $\mathrm{T}_{1}$ 能级升高. 当 $\mathrm{Eu}^{3+} / \mathrm{Tb}^{3+}$ 物 质的量之比为 $7: 1$ 时, $270 \mathrm{~nm}$ 激发下白光 $\mathrm{CIE}$ 坐标为 $(0.34,0.34)$, 显色指数为 $92, \mathrm{CCT}$ 为 $5325 \mathrm{~K}$, 量子产率 为 $5.2 \%$, Bettencourt-Dias 等 ${ }^{[57]}$ 经由蓝光配体 $\mathbf{L 1 3}$ 和 $\mathbf{L 1 4}$ (图 7) 与 $\mathrm{Eu}^{3+} / \mathrm{Tb}^{3+}$ 共掺杂, 制备得到组成分别为 $(\mathbf{L 1 3}) \mathrm{Eu}_{1.8} \mathrm{~Tb}_{28}$ 和 $(\mathbf{L 1 4}) \mathrm{Eu}_{184} \mathrm{~Tb}_{106}$ 的聚合物材料, $270 \mathrm{~nm}$ 激发下白光 $\mathrm{CIE}$ 坐标分别为 $(0.31,0.33)$ 和( $0.38,0.32)$.

李夏等 ${ }^{[33]}$ 利用配体 $\mathrm{H}_{2} \mathbf{L 1 5}$ 和 $\mathbf{L 1 6}$ 与 $\mathrm{La}^{3+}$ 的配位组 装得到二维网状聚合物, $400 \mathrm{~nm}$ 激发下表现出基于配体 中心的蓝光发射. 而 $\mathrm{Eu}^{3+} / \mathrm{Tb}^{3+} / \mathrm{La}^{3+}$ 共掺杂且物质的量 之比为 $18: 2: 80$ 的聚合物在 $370 \mathrm{~nm}$ 激发下发射白光, $\mathrm{CIE}$ 坐标为 $(0.33,0.34), \mathrm{CRI}$ 和 CCT 分别为 88 和 $5658 \mathrm{~K}$. 类似地, Liu 等 ${ }^{[58]}$ 首次以电化学沉积法制备了基于 $\mathrm{H}_{3} \mathbf{L 1 7}$ 与 $\mathrm{Ln}^{3+}$ 的配位聚合物薄膜材料. 当 $\mathrm{Tb}^{3+} / \mathrm{Eu}^{3+}$ 物 质的量之比为 $10: 1$ 时, 组成为 $\mathbf{L 1 7}-\mathrm{Tb}_{10} \mathrm{Eu}_{1}$ 的薄膜材 料在 $350 \mathrm{~nm}$ 激发下发射高纯度白光, 量子产率为 $11.41 \%, \mathrm{CCT}$ 为 $5404 \mathrm{~K}$. 该工作为白光薄膜材料的制备 提供了一种新方法. 杨玉林等 ${ }^{[48]}$ 将吡啶-2,3-二甲酸 $\left(\mathrm{H}_{2} \mathbf{L 1 8}\right)$ 与 $\mathrm{Ln}^{3+}\left(\mathrm{Eu}^{3+}, \mathrm{Tb}^{3+}, \mathrm{Gd}^{3+}\right)$ 的配位聚合物掺杂到 PMMA 中制备得到发光薄膜材料. 当 $\mathrm{Eu}^{3+} / \mathrm{Tb}^{3+} / \mathrm{Gd}^{3+}$ 物 质的量之比为 $0.92: 0.04: 0.04$ 、稀土配位聚合物掺杂 量为 $4 \%$ 时, $285 \mathrm{~nm}$ 激发下得到 CIE 坐标为 $(0.33,0.35)$ 的白光, 苂光寿命为 $1272.26 \mu \mathrm{s}\left(\lambda_{\mathrm{em}}=615 \mathrm{~nm}, \mathrm{Eu}^{3+}\right.$ : $\left.{ }^{5} \mathrm{D}_{0} \rightarrow{ }^{7} \mathrm{~F}_{2}\right)$, 量子产率为 $17.6 \%$.

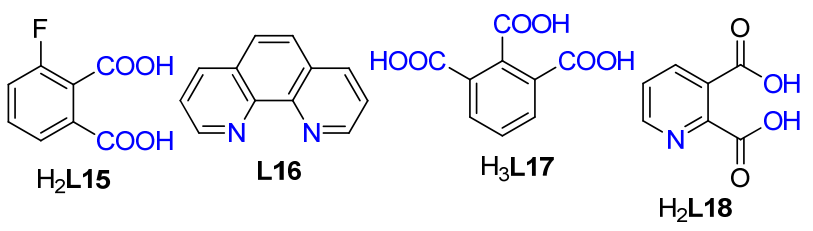

李志强等 ${ }^{[59]}$ 利用三脚架配体 $\mathbf{L 1 9}$ 与 $\mathrm{Ln}^{3+}$ 以 $2: 3$ 的 物质的量之比配位聚合得到光色可调的聚合物. 组成为 $\mathbf{L 1 9}-\mathrm{Eu}_{1} \mathrm{~Tb}_{12}$ 的聚合物在 $375 \mathrm{~nm}$ 激发下发射白光, CIE 坐标为 $(0.33,0.34)$. 研究表明, 激发波长对配体与 $\mathrm{Ln}^{3+}$ 之间的能量传递存在明显的影响, 当激发波长增大时, 能量传递效率降低.

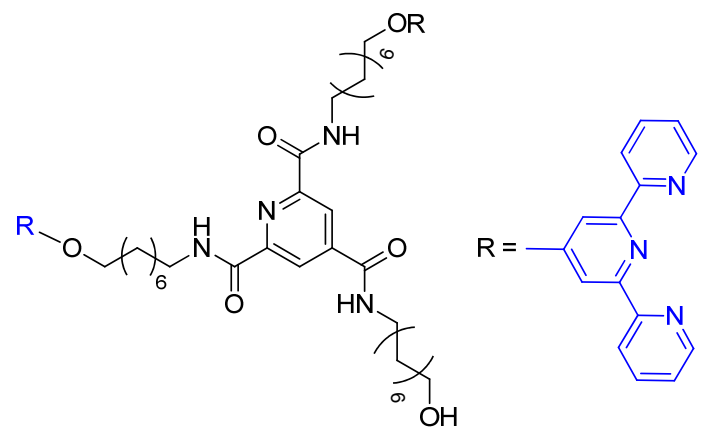

L19
闩鹏飞等 ${ }^{[22}$ 利用 1,8 -薺二酰亚胺的功能化合成了具 有蓝绿光双发射的配体 HL20 和 HL21, 固态下两者发 射波长覆盖 $380 \sim 600 \mathrm{~nm}$. $320 \mathrm{~nm}$ 激发下, 组成为 $\left[\mathrm{Eu}(\mathbf{L 2 0})_{3}\left(\mathrm{CH}_{3} \mathrm{OH}\right)\left(\mathrm{H}_{2} \mathrm{O}\right)\right]_{n}$ 的聚合物发射红光, 而组成 为 $\left\{\left[\mathrm{Eu}(\mathbf{L 2 1})_{3}\left(\mathrm{H}_{2} \mathrm{O}\right)\right] \cdot\left(\mathrm{H}_{2} \mathrm{O}\right)\right\}_{n}$ 的聚合物得到 $\mathrm{CIE}$ 坐标为 $(0.34,0.31)$ 的白光. 值得说明的是, 白光量子产率 $(11.9 \%)$ 高于作者报道的异多核配位聚合物白光材料 ${ }^{[60]}$ 的对应值, 作者认为, 配体 $\mathrm{S}_{1}$ 激发态对 $\mathrm{Eu}^{3+}$ 的敏化发光 使 HL21 的同核聚合物具有较好的发光效率.

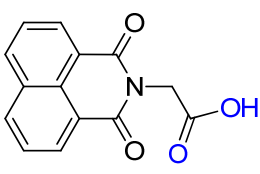

HL20

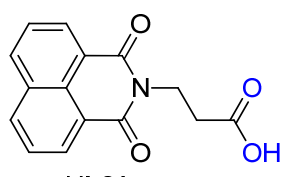

HL21
郑发坤等 ${ }^{[61]}$ 通过水热法制备了基于蓝光配体 $\mathrm{H}_{3} \mathbf{L} 22$ 的光色可调的稀土聚合物材料, 其中组成为 $\left[\mathrm{Sm}(\mathbf{L 2 2})\left(\mathrm{H}_{2} \mathrm{O}\right)_{3}\right] \cdot 5.5 \mathrm{H}_{2} \mathrm{O}$ 的同核聚合物材料在 $358 \mathrm{~nm}$ 激发下, 白光 $\mathrm{CIE}$ 坐标、 CRI、CCT 以及量子产率分别 为 $(0.33,0.31), 87,5748 \mathrm{~K}$ 和 $1.8 \%$. 而 $\mathrm{Eu}^{3+}$ 与 $\mathrm{Gd}^{3+}$ 共掺 杂且组成为 $\left[\left(\mathrm{Eu}_{0.12} \mathrm{Gd}_{0.88}\right)(\mathbf{L 2 2})\left(\mathrm{H}_{2} \mathrm{O}\right)_{3}\right] \cdot 5.5 \mathrm{H}_{2} \mathrm{O}$ 的聚合物 材料在 $360 \mathrm{~nm}$ 激发下得到理想的白光, 白光 $\mathrm{CIE}$ 坐标、 CRI、CCT 以及量子产率分别为 $(0.33,0.33), 5489 \mathrm{~K}, 89$ 和 $1.9 \%$. 童明良等 ${ }^{[62]}$ 通过溶剂热法构筑了基于蓝光配 体 HL23 的光色可调的金属簇, 组成为 $\left[\mathrm{Dy}_{4}(\mathrm{OH})_{2}(\mathbf{L 2 3})_{4}-\right.$ $\left.\left(\mathrm{NO}_{3}\right)_{6}(\mathrm{EtOH})_{2}\right] \cdot \mathrm{EtOH} .335 \mathrm{~nm}$ 激发下白光 $\mathrm{CIE}$ 坐标为 $(0.31,0.32)$, 量子产率为 $2 \% ; 345 \mathrm{~nm}$ 激发下白光肉眼可 见. 有趣的是, 金属簇还具有铁电性、磁性以及手性.
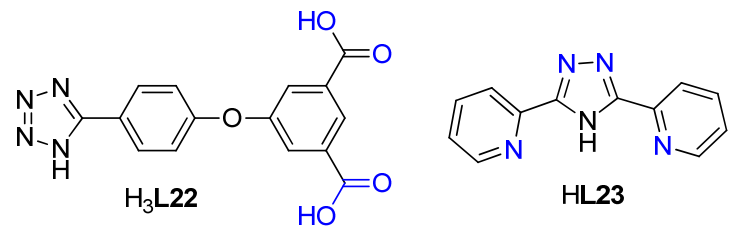

\section{4 白光凝胶}

凝胶媒介给能量给受体对之间提供一个合适的距 离与偶极方向，有利于两者之间的能量转移，同时还能 有效消除因溶剂造成的非辐射衰减 ${ }^{[47]}$. 根据能量给受 体对之间是否配位而有两种凝胶形成机制。一是小分子 有机配体与 $\mathrm{Ln}^{3+}$ 通过配位组装，由此形成的白光凝胶 由于存在较多的非共价弱相互作用，往往具有刺激响应 性 ${ }^{[63]}$; 二是在凝胶基质中分别掺杂蓝光组分(芳香化合 物)和 $\mathrm{Ln}^{3+}{ }^{[64]}$, 两者之间并没有直接配位. 鉴于文献 [47]已对 2017 年前的白光凝胶做了详细的介绍, 在此只 综述 2017 2018 年的相关报道.

Maji 等 ${ }^{[63]}$ 以端基三联吡啶修饰的三脚架配体分子 
L24 为凝胶因子, 在三氯甲烷和四氢呋喃的混合溶液中 与 $\mathrm{Ln}^{3+}$ 配位组装得到金属凝胶. 当 $\mathrm{Eu}^{3+} / \mathrm{Tb}^{3+}$ 物质的量 之比为 $2: 1$ 时凝胶发射白光, CIE 坐标为 $(0.29,0.29)$, 量子产率为 $21.7 \%$. 由于 $\mathrm{Ln}-\mathrm{N}$ 之间的动态非共价作用, 在酸碱、超声以及热刺激响应下能够实现凝胶一溶胶之 间的可逆转变.

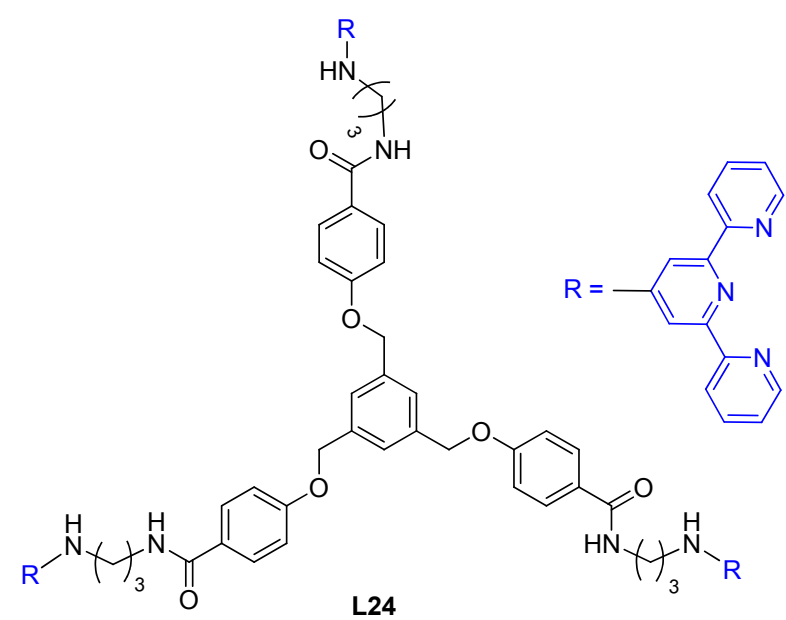

Silva 等 ${ }^{[65]}$ 在亚氨基二乙酸(L25)凝胶因子中掺杂稀 土氧化物得到超分子水凝胶. $\mathrm{pH}$ 为 9 的水溶液中, 当 L25、 $\mathrm{Ln}_{2} \mathrm{O}_{3}$ 的浓度分别为 $0.45,0.075 \mathrm{mmol} \cdot \mathrm{L}^{-1}$, 微波辐 射 $\left(100 \mathrm{~W}, 160{ }^{\circ} \mathrm{C}\right)$ 下形成一系列白光凝胶, 组成分别为 L25- $\mathrm{Eu}_{0.75} \mathrm{~Tb}_{0.25}, \mathbf{L 2 5}-\mathrm{Gd}_{0.7} \mathrm{Eu}_{0.1} \mathrm{~Tb}_{0.2}, \mathbf{L 2 5}-\mathrm{Gd}_{0.7} \mathrm{Eu}_{0.2} \mathrm{~Tb}_{0.1}$, $\mathbf{L 2 5}-\mathrm{Gd}_{0.8} \mathrm{Eu}_{0.05} \mathrm{~Tb}_{0.15}$, 对应的 CIE 坐标依次为 $(0.34$, $0.34),(0.34,0.33),(0.33,0.33)$ 以及 $(0.34,0.33)$.<smiles>O=C(O)CNCC(=O)O</smiles>

Mahapatra 等 ${ }^{[66]}$ 在 $N, N$-二甲基甲酰胺溶液中分别通 过配体 L26 和 L27 分子间的 $\pi-\pi$ 堆积以及氢键作用 $(\mathrm{C}$ $-\mathrm{H} \cdots \mathrm{F} / \mathrm{C}-\mathrm{H} \cdots \mathrm{Cl})$ 构筑了超分子凝胶, 进而与 $\mathrm{Tb}^{3+} / \mathrm{Eu}^{3}$ +配位组装得到红光和绿光发射的金属凝胶. 当配体与 $\mathrm{Tb}^{3+}$ 和 $\mathrm{Eu}^{3+}$ 物质的量之比为 $1: 0.5: 0.5$ 时调制得到近 白光发射 $\left(\lambda_{\mathrm{ex}}=285 \mathrm{~nm}\right)$ 的金属凝胶. 其中配体 $\mathbf{L 2 6}$ 形成 的白光凝胶 CIE 坐标为 $(0.31,0.36), \mathbf{L} 27$ 对应的 CIE 坐标 为 $(0.34,0.36)$.

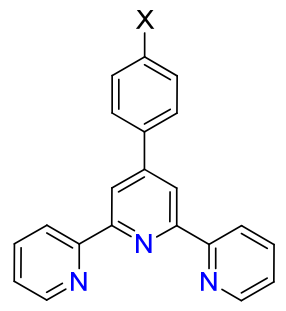

L26: $X=F ; L 27: X=C I$

\section{5 稀土金属有机框架白光材料}

由于配体结构的多样性以及孔道尺寸可调的优势, LnOFs 材料无疑是调制白光最有潜力的多孔材料 ${ }^{[10]}$. 配 体分子既是 $\mathrm{LnOFs}$ 材料的构筑模块也是 $\mathrm{Ln}^{3+}$ 的敏化基 才，其中配体与 $\mathrm{Ln}^{3+}$ 之间的配位作用以及配体分子间 的氢键、 $\pi-\pi$ 堆积等弱相互作用都有助于 LnOFs 刚性的 增强, 而被配体隔离的 $\mathrm{Ln}^{3+}$ 能够防止荧光的自猝灭 ${ }^{[13]}$, 因而 LnOFs 白光材料普遍具有较好的热稳定性以及较 高的光量子产率. LnOFs 白光材料目前主要有两种构筑 方法, 一是通过多齿配体与不同光色的 $\mathrm{Ln}^{3+}$ 配位聚合; 二是通过 $\mathrm{d}-$ 区金属框架 $(\mathrm{MOF})$ 为主体材料封装不同光色 的 $\mathrm{Ln}^{3+}$ 并经由 MLCT 激发态敏化发光 ${ }^{[62,67]}$. 通过 $\mathrm{Ln}^{3+}$ 嵌入量、激发波长以及温度等条件的调控, 使 LnOFs 光 色可调并调制白光. 在此, 按照 LnOFs 中 $\mathrm{Ln}^{3+}$ 种类的不 同，分别介绍异多核和同核 LnOFs 白光材料.

\section{1 异多核 LnOFs 白光材料}

异多核 LnOFs 白光材料中存在两种及两种以上金 属离子, 至少有一种为 $\mathrm{Ln}^{3+}$, 最常见到的 $\mathrm{Ln}^{3+}$ 组合方式 有 $\mathrm{Eu}^{3+} / \mathrm{Tb}^{3+}$ 和 $\mathrm{Eu}^{3+} / \mathrm{Tb}^{3+} / \mathrm{La}^{3+}\left(\mathrm{Gd}^{3+}\right)$. 如张健等 ${ }^{[50]}$ 以 $\mathrm{H}_{3} \mathbf{L} 28$ 与 $\mathrm{Cu}^{2+}$ 配位组装形成的三维 $\mathrm{MOF}$ 为主体材料, 经由液相外延生长技术封装稀土配合物 Ln-3L29 $\left(\left[\mathrm{Me}_{2} \mathrm{NH}_{2}\right]_{3}\left[\mathrm{Cd}_{0.9365} \mathrm{Eu}_{0.0370} \mathrm{~Tb}_{0.0265}(\mathbf{L 2 9})_{3}\right]\right)$. 当 $\mathrm{Eu}^{3+} / \mathrm{Tb}^{3+} /$ $\mathrm{Gd}^{3+}$ 物质的量之比比为 $0.0131: 0.830: 0.157$ 时, 291 $\mathrm{nm}$ 激发下得到 CIE 坐标为 $(0.37,0.38)$ 的白光, 量子产率 为 $46.50 \%, \mathrm{CRI}$ 和 CCT 分别是 82.8 和 $5880 \mathrm{~K}$. 虽然该 工作为白光材料的制备提供了一种新方法, 但与未封装 之前相比 ${ }^{[41]}$, 量子产率明显减小.<smiles>O=C(O)c1cc(C(=O)O)cc(C(=O)O)c1</smiles>

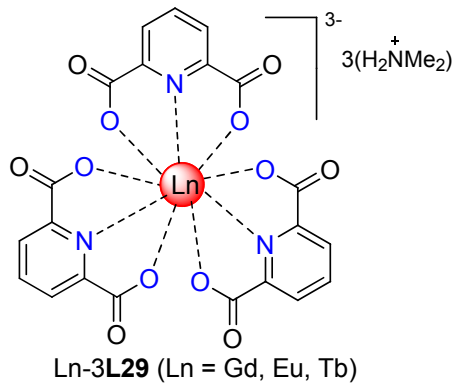

王州课题组 ${ }^{[31]}$ 利用配体 $\mathbf{L 2 5}$ 构筑了 $\mathrm{LnOFs}$ 材料, 组成为 $\mathrm{Gd}_{99} \mathrm{Eu}_{0.5} \mathrm{~Tb}_{0.5}$ 时, $365 \mathrm{~nm}$ 激发下可得到 $\mathrm{CIE}$ 坐标 为 $(0.30,0.38)$ 的白光材料. 作者研究了组成为 $\mathrm{Gd}_{99} \mathrm{~Tb}_{0.1}$ $\mathrm{Eu}_{0.9}$ 的 $\mathrm{LnOF}$ 材料在不同剂量紫外线(365 nm)辐射下对 CIE 坐标的影响, 其中辐射剂量为 $4.590 \mathrm{~mJ}$ 时得到纯白 光发射.

熊克才等 ${ }^{[68]}$ 将 $\mathrm{Eu}^{3+}, \mathrm{Tb}^{3+}, \mathrm{Dy}^{3+}$ 嵌入到配体 $\mathrm{H}_{4} \mathbf{L 3 0}$ 的 $N, N$-二乙基甲酰胺 (DEF) 水溶液中得到组成为 $\mathrm{Ln}(\mathrm{HL30})\left(\mathrm{H}_{2} \mathrm{O}\right)(\mathrm{DEF})$ 的 LnOFs 材料. 通过调节三种 
$\mathrm{Ln}^{3+}$ 的化学计量比、改变激发波长以及控制水热法合成 温度的策略成功得到白光材料. $\mathrm{Ln}^{3+}$ 的组成为 $\mathrm{Eu}_{0.0667^{-}}$ $\mathrm{Tb}_{0.0667} \mathrm{Dy}_{0.8666}$, 激发波长为 $336 \mathrm{~nm}$ 时, 白光色纯度与温 度的变化相关. 温度为 100 和 $50 \mathrm{~K}$ 时, CIE 坐标分别为 $(0.32,0.25)$ 和 $(0.30,0.32)$. 当 $\mathrm{Ln}^{3+}$ 的组成为 $\mathrm{Eu}_{0.0666^{-}}$ $\mathrm{Tb}_{0.4667} \mathrm{Dy}_{0.4667}$ 、温度为 200 和 $150 \mathrm{~K}$ 时, CIE 坐标分别为 $(0.31,0.25)$ 和 $(0.30,0.30)$. 可见, 在 $\mathrm{Ln}^{3+}$ 浓度或化学计 量比一定时, 在合理的温度变化范围内, 温度的下降有 利于白光色纯度的提高. 赵显等 ${ }^{[69]}$ 同样利用配体 $\mathrm{H}_{4} \mathbf{L 3 0}$ 制备了 $\mathrm{Eu}, \mathrm{Dy}$ 共掺杂的白光材料.

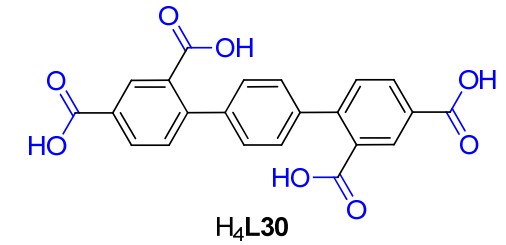

吴大雨等[70]经由配体 $\mathbf{L 3 1}$ 和 $\mathbf{L 3 2}$ 与 $\mathrm{Zn}^{2+}$ 配位组装
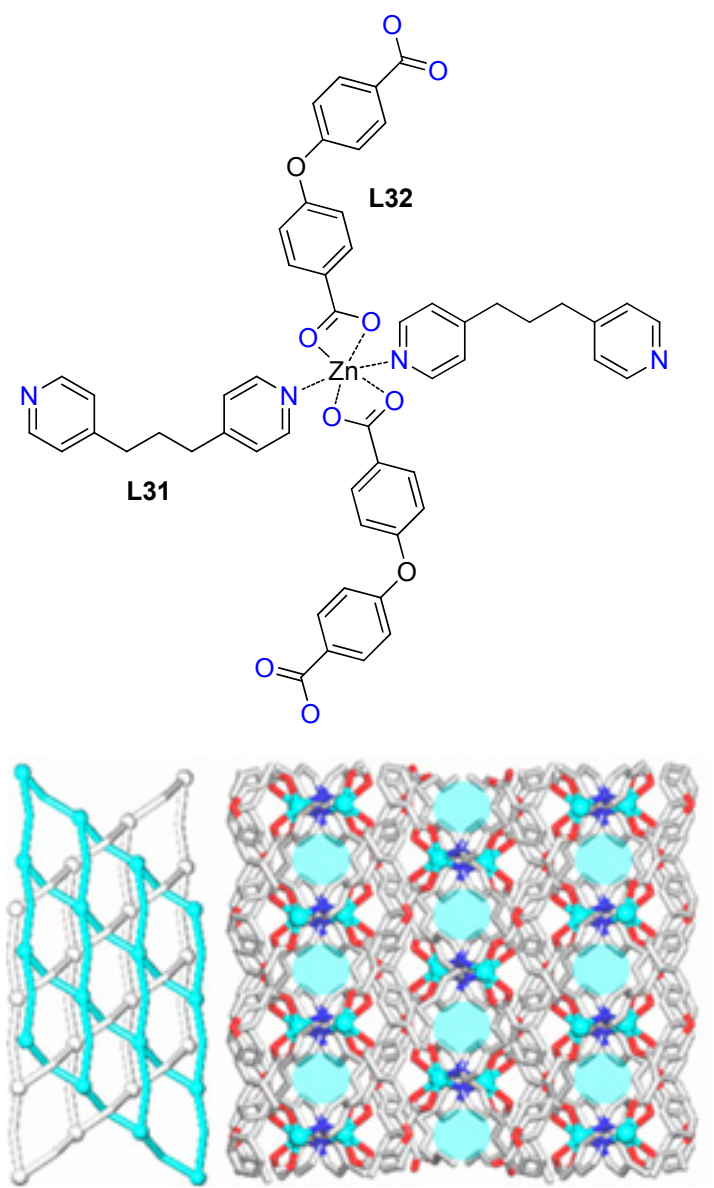

图 $8 \mathrm{L31}$ 和 $\mathbf{L 3 2}$ 与 $\mathrm{Zn}^{2+}$ 的组装模式以及 $\mathrm{Zn}-\mathrm{MOF}$ 的平面 $(2 \mathrm{D}+2 \mathrm{D})$ 互穿结构片段

Figure 8 Assembly model between mixed ligands (L31 and L32) and $\mathrm{Zn}^{2+}$ and the structural fragments of $2 \mathrm{D}+2 \mathrm{D}$ interpenetrated layers
构筑了二维平面互穿的 $\operatorname{MOF}$ (图 8). 以蓝光发射的 $\mathrm{Zn}-\mathrm{MOF}$ 为主体框架, 封装 $\mathrm{Tb}^{3+} / \mathrm{Eu}^{3+}$ 后形成发光可调的 固态发光材料. 当 $\mathrm{Tb}^{3+} / \mathrm{Eu}^{3+}$ 的物质的量之比为 $47: 9$ 时, $300 \mathrm{~nm}$ 激发下白光 CIE 坐标为 $(0.33,0.32)$, 但量子 产率仅为 $2.8 \%$, 在水溶液中的苂光寿命为 $463 \mu \mathrm{s}$ $\left(\lambda_{\mathrm{em}}=545 \mathrm{~nm}, \mathrm{~Tb}^{3+}:{ }^{5} \mathrm{D}_{4} \rightarrow{ }^{7} \mathrm{~F}_{5}\right)$. 刘志亮等 ${ }^{[67]}$ 构筑了组成 为 $\left.\left[\mathrm{Zn}\left(\mathrm{H}_{2} \mathbf{L} 33\right)_{0.5}(\mathbf{L 3 4})\right] \cdot 5 \mathrm{H}_{2} \mathrm{O}\right]_{n}$ 的蓝光 $\mathrm{MOF}$ 材料. MOF 孔道中未参与配位的羧基进一步与 $\mathrm{Eu}^{3+}$ 和 $\mathrm{Tb}^{3+}$ 配位, 控制 $\mathrm{Eu}^{3+} / \mathrm{Tb}^{3+}$ 物质的量之比为 $3: 1$ (实测值为 $6.8: 2.2$ ) 时, $260 \mathrm{~nm}$ 激发下白光 $\mathrm{CIE}$ 坐标为 $(0.34,0.35)$, 量子产 率仅为 $1 \%$, 在不同发射波长下的荧光寿命分别为 1.45 $\mathrm{ms}\left(\lambda_{\mathrm{em}}=545 \mathrm{~nm}, \mathrm{~Tb}^{3+}:{ }^{5} \mathrm{D}_{4} \rightarrow{ }^{7} \mathrm{~F}_{5}\right)$ 和 $2.76 \mathrm{~ms}\left(\lambda_{\mathrm{em}}=618\right.$ $\left.\mathrm{nm}, \mathrm{Eu}^{3+}:{ }^{5} \mathrm{D}_{0} \rightarrow{ }^{7} \mathrm{~F}_{2}\right)$. 臓双全等 ${ }^{[71]}$ 以配体 $\mathrm{H}_{5} \mathbf{L 3 5}$ 与 $\mathrm{Zn}^{2+}$ 的 MOF 材料为主体框架, 封装 $\mathrm{Eu}^{3+} / \mathrm{Tb}^{3+}$ 后得到白光材 料, 当两者物质的量之比分别为 $0.127: 0.432$ 以及 $0.183: 0.408$ 时, 白光材料的 CIE 坐标分别为( 0.31 , $0.34)$ 和 $(0.34,0.33)$, 白光量子产率分别为 $7 \%$ 和 $8 \%$. Holdt $^{[72]}$ 、兰亚乾 ${ }^{[73]}$ 和孙福兴 ${ }^{[74]}$ 等课题组分别通过 $\mathrm{Zn}-\mathrm{MOF}$ 主体材料封装 $\mathrm{Ln}^{3+}$ 的策略调制了白光.

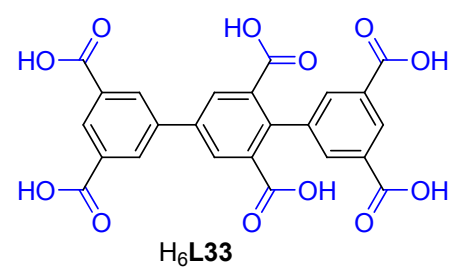<smiles>c1c[nH]c(-c2cc(-c3ncc[nH]3)cc(-c3ncc[nH]3)c2)n1</smiles><smiles>CCCCCCCCC(=O)c1cc(COc2cc(C(=O)O)cc(C(=O)O)c2)cc(C(=O)O)c1</smiles>

杨玉林等 ${ }^{[75]}$ 通过 $H_{2} \mathbf{L} 36$ 分子间的氢键和 $\pi-\pi$ 堆积以 及与硝酸铟的配位作用，在水溶液中构筑了蓝光发射的 MOF, 进而与 $\mathrm{Eu}^{3+} / \mathrm{Dy}^{3+}$ 配位形成 LnOFs 材料. 研究表 明，随着 $\mathrm{Eu}^{3+}$ 浓度的增加(0 到 $\left.1.6 \mu \mathrm{mol} \cdot \mathrm{L}^{-1}\right), \mathrm{LnOFs}$ 光 色从蓝色转变为红色. 控制两种离子的掺杂量 $\left(\mathrm{Dy}_{0.87} \mathrm{Eu}_{0.13}\right)$ 即可得到白光发射, CIE 坐标为 $(0.33,0.33)$. 在此过程中实现了对 $\mathrm{Eu}^{3+}$ 的快速高灵敏度的检测(最低 检出限为 $\left.0.87 \mu \mathrm{mol} \cdot \mathrm{L}^{-1}\right)$. 杜少武等 ${ }^{[76]}$ 利用组成为 $\mathrm{Cd}_{2} \mathrm{~K}_{4}(\mathbf{L 1 8})_{4}$ 的蓝绿光 $\mathrm{MOF}$ 为主体框架, 通过 $\mathrm{Eu}^{3+}$ 掺杂 量和激发波长的改变, 调制得到一系列白光材料.

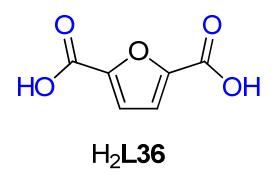


侯磊等 ${ }^{[77]}$ 以共轭的 $N, N^{\prime}$-二(3,5-二羧基苯基)草酰胺 $\left(\mathrm{H}_{4} \mathbf{L}\right.$ 37) 为能量给体, 在 $N, N^{\prime}$-二甲基乙酰胺(DMA)、乙 腈和水的混合溶剂中与 $\mathrm{Ln}^{3+}$ 配位聚合构筑了三种组成 为 $\left\{\left[\mathrm{Ln}_{2}(\mathbf{L 3 6})_{1.5}(\mathrm{DMA})_{3}\left(\mathrm{H}_{2} \mathrm{O}\right)\right] \cdot 5 \mathrm{H}_{2} \mathrm{O}\right\}_{n}\left(\mathrm{Ln}=\mathrm{Eu}^{3+}, \mathrm{Gd}^{3+}\right.$, $\left.\mathrm{Tb}^{3+}\right)$ 的 LnOFs 材料. 当 $\mathrm{Eu}^{3+} / \mathrm{Gd}^{3+} / \mathrm{Tb}^{3+}$ 共掺杂且物质的 量之比为 $0.0855: 0.6285: 0.2860$ 时, $390 \mathrm{~nm}$ 激发下得 到 CIE 坐标为 $(0.34,0.33)$ 的白光, 量子产率为 $22.4 \%$, $\mathrm{CCT}$ 为 $5219 \mathrm{~K}$.<smiles>O=C(Nc1cc(C(=O)O)cc(C(=O)O)c1)C(=O)Nc1cc(C(=O)O)cc(C(=O)O)c1</smiles>

李夏等 ${ }^{[32,40,78 ~ 80]}$ 利用小分子配体 $\left(\mathbf{L 1 6}, \mathrm{H}_{2} \mathbf{L 3 8}, \mathbf{L 3 9}\right.$, $\mathrm{H}_{2} \mathbf{L} 40, \mathrm{H}_{2} \mathbf{L} 41, \mathrm{H}_{2} \mathbf{L} 42, \mathbf{L 4 3}, \mathbf{L 4 4}$ )制备了一系列异多核 LnOFs 白光材料. 最具代表性的是组成为 $\mathrm{Gd}_{0.91} \mathrm{Eu}_{0.05^{-}}$ $\mathrm{Tb}_{0.04}(\mathbf{L 4 0}) \mathbf{L 1 6}(\mathbf{L 4 1})_{0.5}$ 的 LnOFs 材料, 其在 $370 \mathrm{~nm}$ 激发 下白光 CIE 坐标、CCT 以及 CRI 分别为 $(0.33,0.34) 、 5454$ $K$ 和 84. 值得一提的是, 由于辅助配体 $\mathrm{H}_{2} \mathbf{L} \mathbf{4 1}$ 阻止了溶 剂水分子对 $\mathrm{Ln}^{3+}$ 的竞争结合, 从而有效改善了发光效率 (白光量子产率为 $38.7 \%$ ). 另外, 组成为 $\left[\left(\mathrm{Yb}_{0.73} \mathrm{~Tb}_{0.25}\right.\right.$ $\left.\left.\mathrm{Eu}_{0.02}\right)_{2}(\mathbf{L 4 2})_{2}(\mathbf{L 1 6})_{2}(\mathbf{L 4 1})\left(\mathrm{H}_{2} \mathrm{O}\right)\right]$ 的 $\mathrm{LnOF}$ 材料在 $355 \mathrm{~nm}$ 激发下, 上述相关参数依次为 $(0.35,0.36), 4934 \mathrm{~K}, 89$ 和 $20.4 \%$.

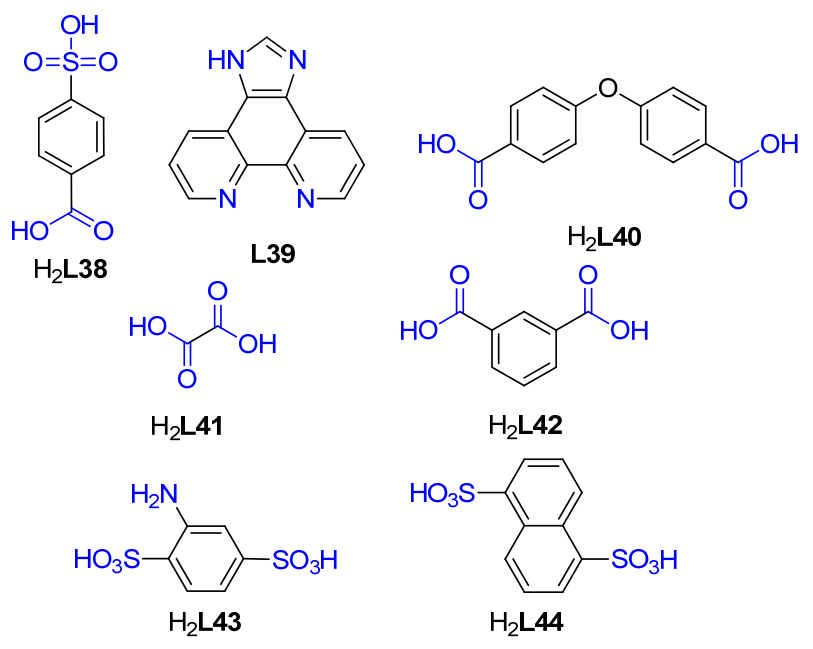

Rodrigues 等 ${ }^{[81]}$ 通过配体 $\mathrm{H}_{6} \mathbf{L} \mathbf{4 5}$ 与 $\mathrm{Ln}^{3+} \mathrm{Eu}^{3+}, \mathrm{Tb}^{3+}$, $\mathrm{Gd}^{3+}$ 的配位组装合成了三种发光材料, 进而利用逐层外 延生长技术制备了 EuTbGd，EuGdTb，GdEuTb 以及 $\mathrm{GdTbEu}$ 四种 $\mathrm{LnOFs}$ 白光材料四种 $\mathrm{LnOFs}$ 白光材料, 分 别在 $361,347,378$ 和 $360 \mathrm{~nm}$ 激发下, CIE 坐标依次为 $(0.34,0.34),(0.34,0.33),(0.34,0.34)$ 以及 $(0.33,0.34)$.<smiles>CCCCCC(=O)OC(=O)c1c(C(=O)O)c(C(=O)O)c(C(=O)O)c(C(=O)O)c1C(=O)O</smiles>

张洪杰等 ${ }^{[82]}$ 经由配体 $\mathrm{H}_{4} \mathbf{L} \mathbf{4 6}$ 构筑了光色可调的三 维 $\mathrm{LnOF}$ 材料, 其组成为 $\left[\mathrm{LnL} \mathbf{4 6}\left(\mathrm{H}_{2} \mathrm{O}\right)_{3}\right] \cdot 2 \mathrm{H}_{2} \mathrm{O}$. 当 $\mathrm{Dy}^{3+}$ / $\mathrm{Eu}^{3+}$ 或 $\mathrm{Sm}^{3+} / \mathrm{Dy}^{3+}$ 分别与 $\mathrm{Gd}^{3+}$ 共掺杂且在 $293 \mathrm{~nm}$ 激发 下得到一系列近白光发射的材料, 色坐标显示色纯度并 不是很好. 遗憾的是作者并没有详细考察白光 CCT、 CRI 以及量子产率.

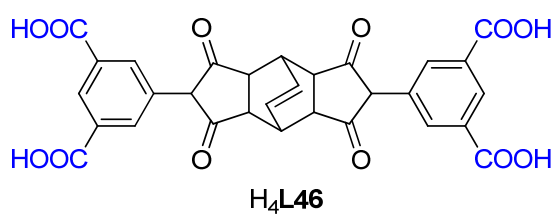

王莉等 ${ }^{[83]}$ 利用配体 $\mathrm{H}_{2} \mathbf{L} \mathbf{4 7}$ 构筑了一系列 LnOFs 材 料, 晶体结构表明, 配体间的 $\pi-\pi$ 堆积以及配体与 $\mathrm{Ln}^{3+}$ 的配位组装使其形成一维双链结构. 当 $\mathrm{Eu}^{3+} / \mathrm{Gd}^{3+} / \mathrm{Tb}^{3+}$ 共掺杂时, $365 \mathrm{~nm}$ 激发下均可得到近白光发射, 尤其是 当物质的量之比为 $0.25: 0.50: 0.25$ 时，白光 $\mathrm{CIE}$ 坐标 为 $(0.34,0.33)$, 量子产率为 $9.75 \%$. 钱国栋等 ${ }^{[84]}$ 在 $\mathrm{Gd}^{3+}$ 与 $\mathrm{H}_{2} \mathbf{L} \mathbf{4 7}$ 的主体 MOF 中掺杂摩尔分数为 $1.0 \%$ 和 $2.5 \%$ 以及 $1.5 \%$ 和 $3.0 \%$ 的 $\mathrm{Eu}^{3+}$ 和 $\mathrm{Tb}^{3+}, 330 \mathrm{~nm}$ 激发下 $\mathrm{CIE}$ 坐 标分别为 $(0.34,0.33)$ 和 $(0.34,0.34)$. 王莉等 ${ }^{[85]}$ 通过 $\mathrm{H}_{2} \mathbf{L 4 8}$ 构筑了组成为 $\left[\left(\mathrm{Eu}_{0.0073} \mathrm{~Tb}_{0.0007} \mathrm{Gd}_{0.992}\right)_{2}\left(\mathrm{H}_{2} \mathrm{O}\right)_{4^{-}}\right.$ $\left.(\mathbf{L 4 8})_{3}\right] \cdot 2 \mathrm{H}_{2} \mathrm{O}$ 的 $\mathrm{LnOF}$ 材料, $340 \mathrm{~nm}$ 激发下白光 $\mathrm{CIE}$ 坐 标为 $(0.34,0.33)$.<smiles>O=C(O)c1cc(C(=O)O)cc(-c2nn[nH][13c]2-c2ccccc2)c1</smiles>

$\mathrm{H}_{2} \mathrm{~L} \mathbf{}$ 7<smiles>O=C(O)c1cc(O)cc(C(=O)O)n1</smiles>

$\mathrm{H}_{2} \mathrm{~L} 48$
杜少武等 ${ }^{[86]}$ 报道了组成为 $\left[\left(\mathrm{Eu}_{0.004} \mathrm{~Tb}_{0.046} \mathrm{Gd}_{0.950)}\right)\right.$ (L49)(DMSO) $\left.)_{2}\right] \cdot \mathrm{H}_{2} \mathrm{O}$ 的异多核 LnOF 材料. 研究表明, 其光色具有激发波长的响应性. 激发波长为 $315 \mathrm{~nm}$ 时, 配体与 $\mathrm{Ln}^{3+}$ 之间有效的能量转移使其发射黄光; 而在 $365 \mathrm{~nm}$ 激发下, 由于配体 $\mathrm{S}_{1}$ 与 $\mathrm{T}_{1}$ 激发态之间不能进行 有效的系间窝跃，因而 LnOF 只能发射基于配体中心的 蓝光; 只有在 $347 \mathrm{~nm}$ 激发时, $\mathrm{LnOF}$ 发射白光, $\mathrm{CIE}$ 坐标 为 $(0.36,0.32)$. 有趣的是, 通过改变激发波长的方法, 可实现光色从黄到白再到蓝光的可逆转变. 
<smiles>CCCCCC(=O)c1ccc(-c2cc(-c3ccc(C(=O)O)cc3)cc(-c3ccc(C(=O)O)cc3)c2)cc1</smiles>

刘术侠等 ${ }^{[87]}$ 报道了组成为 $\left[\mathrm{Ln}(\mathbf{L 5 0}) \mathrm{H}_{2} \mathrm{O}\right] \cdot 2 \mathrm{DMF}$ • $3 \mathrm{H}_{2} \mathrm{O}$ 的 $\mathrm{LnOFs}$ 材料. 当 $\mathrm{Eu}^{3+} / \mathrm{Tb}^{3+}$ 的物质的量之比为 0.5 : 99.5 时, $365 \mathrm{~nm}$ 激发下白光量子产率高达 $46.15 \%$, $\mathrm{CCT}$ 为 $5818 \mathrm{~K}, \mathrm{CIE}$ 坐标为 $(0.33,0.33)$. 另外, 当 $\mathrm{La}^{3+}$ / $\mathrm{Eu}^{3+} / \mathrm{Tb}^{3+}$ 的物质的量之比为 $0.6: 0.1: 0.3$ 时, $365 \mathrm{~nm}$ 激 发下白光量子产率为 $47.33 \%, \mathrm{CIE}$ 坐标为 $(0.32,0.32)$, $\mathrm{CCT}$ 为 $6370 \mathrm{~K}$.<smiles>CCCCCCCCCCCCCCCCCCCC(=O)O</smiles>

间冰等 ${ }^{[88]}$ 利用配体 $\mathrm{H}_{3} \mathbf{L 5 1}$ 和 $\mathrm{Al}^{3+}$ 形成的多孔纳米 微晶作为主体框架, 封装 $\mathrm{Ln}^{3+}$ 后得到纳米级的 $\mathrm{LnOF}$ 材 料. 光学研究表明纳米微晶发射配体苂光(421 nm), 当 掺杂 $\mathrm{Eu}^{3+}$ (质量分数为 $1.25 \times 10^{-7}$ ) 后, $350 \mathrm{~nm}$ 激发下白 光 $\mathrm{CIE}$ 坐标为 $(0.33,0.25)$, 量子产率介于 $15 \% \sim 19 \%$. 该课题组 ${ }^{[89]}$ 以类似的方法制备了基于配体 $\mathrm{H}_{2} \mathbf{L 5 2}$ 和 $\mathrm{Zr}^{4+}$ 的 MOF 材料. 在此主体材料中封装 $\mathrm{Eu}^{3+}$ 离子后, 分 别在 370,380 以及 $390 \mathrm{~nm}$ 激发下得到 CIE 坐标依次为 $(0.43,0.36),(0.34,0.34)$ 和 $(0.31,0.33)$ 的白光. 另外, 该 课题组 ${ }^{[90]}$ 在 $\mathrm{H}_{2} \mathbf{L 5 2}$ 与 $\mathrm{Al}^{3+}$ 形成的 MOF 中封装 $\mathrm{Eu}^{3+}$ 及辅 助配体 TTA 之后得到白光聚合物材料, 并以此为 LED 芯片涂层制备了 WLED, 光色与理想白光几乎相同. 白 凤英 等 $^{[91]}$ 的近期工作同样证明, 白光可通过蓝光和红 光调制而成.<smiles>O=C(O)c1ccc(C(=O)O)c(C(=O)O)c1</smiles>

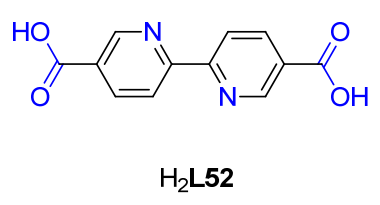

张献明等 ${ }^{[27]}$ 报道了两种基于 $\mathrm{H}_{3} \mathbf{L 5 3}$ 的异多核
LnOFs 材料, 组成为 $\mathrm{Eu}_{0.045} \mathrm{~Tb}_{0.955} \mathbf{L 5 3}$ 时, $365 \mathrm{~nm}$ 激发下 白光 CIE 坐标为 $(0.33,0.32)$, 光量子产率为 $15 \%$. 作者 以此白光材料为涂层成功制备了以蓝光为光源的 WLED, CIE 坐标为 $(0.33,0.34)$, CCT 以及 CRI 分别为 $5733 \mathrm{~K}$ 和 73.4. 组成为 $\mathrm{La}_{0.6} \mathrm{Eu}_{0.1} \mathrm{~Tb}_{0.3} \mathrm{L53}$ 的 $\mathrm{LnOF}$ 材料 在 $365 \mathrm{~nm}$ 激发下, 白光 CIE 坐标为 $(0.32,0.33)$, 光量子 为 $14.4 \%$.<smiles>O=C(O)c1ccc(COc2cc(OCc3ccc(C(=O)O)cc3)cc(C(=O)O)c2)cc1</smiles>

胡怀明等 ${ }^{[39]}$ 利用配体 HL54 和 $\mathrm{H}_{2} \mathbf{L 5 5}$ 与 $\mathrm{Ln}^{3+}$ 配位 组装得到光色可调的 LnOF 材料, 其中桥联配体 $\mathrm{H}_{2} \mathbf{L 5 5}$ 的存在能够有效阻止水分子与稀土离子结合的结合, 进 而提高发光材料的发光效率. 在水热合成条件下, 精确 控制反应酸度 $(\mathrm{pH}=4)$ 以及 $\mathrm{Eu}^{3+} / \mathrm{Tb}^{3+}$ 的物质的量之比为 $4: 6$ 时, $405 \mathrm{~nm}$ 激发下白光 $\mathrm{CIE}$ 坐标为 $(0.32,0.34)$, 光 量子产率为 $6.03 \%$. 有趣的是, 这种发光材料对温度具 有很好的传感能力.<smiles>O=S(=O)(O)c1ccccc1-c1nc2c3cccnc3c3ncccc3c2[nH]1</smiles>

HL54<smiles>O=C(O)CCCC(=O)O</smiles>

$\mathrm{H}_{2} \mathrm{~L} 55$
吴克琛等 ${ }^{[92]}$ 通过水热法构筑了基于 L56、 $\mathrm{H}_{2} \mathbf{L 4 2}$ 和 $\mathrm{La}^{3+}$ 的 LnOF 材料, 在 $350 \sim 400 \mathrm{~nm}$ 激发下因蓝光和黄 光双发射而得到白光, $390 \mathrm{~nm}$ 激发下 CIE 坐标为( 0.27 , $0.32), \mathrm{CRI}$ 为 $71, \mathrm{CCT}$ 为 $10068 \mathrm{~K}, 370 \mathrm{~nm}$ 激发下量子产 率为 $11 \%$. 当在这种主体框架中掺杂 $\mathrm{Eu}^{3+}(\mathrm{Eu} / \mathrm{La}$ 物质 的量之比不超过 $0.0016: 1$ )且在 $390 \mathrm{~nm}$ 激发下, CRI, $\mathrm{CCT}$ 以及 $\mathrm{CIE}$ 坐标基本与理想白光的指标一致. 其中 $\mathrm{Eu} / \mathrm{La}$ 物质的量之比为 $0.001: 1$ 时量子产率为 $12 \%$.

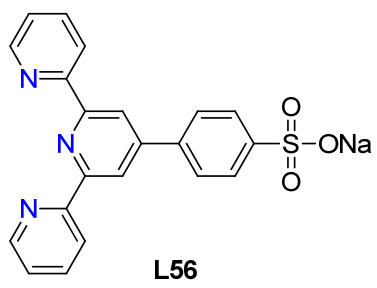

\section{2 同核 LnOFs 白光材料}

显然，同核 LnOFs 白光材料中只存在一种金属离子 且为 $\mathrm{Ln}^{3+}$. 结合 “光色互补原则”, 经由单/同核配合 
物/聚合物调制白光时, 白光通常由配体蓝绿光双发 射 ${ }^{[22,36]}$ 与 $\mathrm{Ln}^{3+}$ 的红光或配体蓝光与 $\mathrm{Ln}^{3+}$ 的多种光色(或 黄光)复合而成. $\mathrm{Sm}^{3+}$ 和 $\mathrm{Dy}^{3+}$ 是单/同核稀土配合物/聚合 物白光材料中最常用的 $\mathrm{Ln}^{3+}$, 前者分别在 560 (绿光)、 598(黄光)和 640 (红光) $\mathrm{nm}$ 处存在发射; 而 $\mathrm{Dy}^{3+}$ 发射蓝光 $(480 \mathrm{~nm})$ 、黄光 $(573 \mathrm{~nm})$ 和红光 $(661 \mathrm{~nm})$, 但遗憾的是两 者不符合 “能量匹配原则” 而很难被敏化发光 ${ }^{[5]}$, 导致 白光量子产率较低. 如李夏等 ${ }^{[80}$ 基于 $\mathrm{Sm}^{3+}$ 的特征发射, 构筑了组成为 $\left\{\left[\mathrm{Sm}_{2}(\mathbf{L 4 9})_{3}(\mathbf{L 3 9})_{4}\left(\mathrm{H}_{2} \mathrm{O}\right)_{2}\right] \cdot 9 \mathrm{H}_{2} \mathrm{O}\right\}_{n}$ 的白光 LnOFs 材料, $390 \mathrm{~nm}$ 激发下白光 CIE 坐标为 $(0.33,0.32)$, $\mathrm{CCT}$ 和 CRI 分别为 $5722 \mathrm{~K}$ 和 84 , 而白光量子产率并没 有报道. 另外, 李光明等 ${ }^{[93]}$ 通过羧酸配体 $\mathrm{H}_{2} \mathbf{L 5 7}$ 构筑了 组成为 $\left\{\left[\mathrm{Sm}(\mathbf{L 5 7})_{1.5}\left(\mathrm{H}_{2} \mathrm{O}\right)_{2}\right] \cdot \mathrm{H}_{2} \mathrm{O}\right\}_{n}$ 的二维 $\mathrm{LnOF}$ 材料, $365 \mathrm{~nm}$ 激发下白光 $\mathrm{CIE}$ 坐标为 $(0.33,0.33), \mathrm{CCT}$ 为 5459 $\mathrm{K}$, 量子产率仅为 $0.73 \%$. 经由配体 $\mathrm{H}_{2} \mathbf{L 5 8}$ 与 $\mathrm{Sm}^{3+}$ 所构 筑的 $\mathrm{LnOF}$ 材料在 $400 \mathrm{~nm}$ 激发下仅得到近白光发射 ${ }^{[94]}$, $\mathrm{CIE}$ 坐标为 $(0.31,0.23)$.
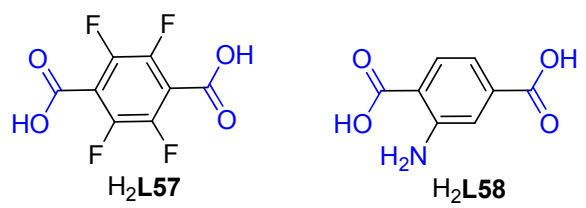

间冰等 ${ }^{[88}$ 的工作突破了 “光色互补原则” 的限制, 经由蓝光和 $\mathrm{Eu}^{3+}$ 红光调制白光, 但白光色纯度相对较 差. 吴克琛等 ${ }^{[95]}$ 通过蓝绿光发射的共轭配体 $\mathrm{H}_{2} \mathbf{L} 59$ 和 $\mathrm{Eu}^{3+}$ 的配位组装得到组成为 $\left[\mathrm{Eu}\left(\mathrm{H}_{2} \mathrm{O}\right)_{2}(\mathrm{OH})(\mathrm{HL59})\right]$ $\mathrm{H}_{2} \mathrm{O}$ 的二维 $\mathrm{LnOF}$ 材料, 由于高能 $\mathrm{O}-\mathrm{H}$ 键振动造成的 非辐射衰减使其只表现出较弱的红光发射. 当存在辅助 配体 $\mathrm{H}_{2} \mathbf{L} 41$ 时得到三维 $\mathrm{LnOF}$ 材料, 组成为 $\left[\mathrm{Eu}\left(\mathrm{H}_{2} \mathrm{O}\right)(\mathbf{L 4 1}) 0.5(\mathrm{HL59})\right] \cdot 2 \mathrm{H}_{2} \mathrm{O}, 380 \mathrm{~nm}$ 激发下获得 $\mathrm{CIE}$ 坐标为 $(0.31,0.35)$ 的近白光发射, 量子产率为 $9.2 \%$.

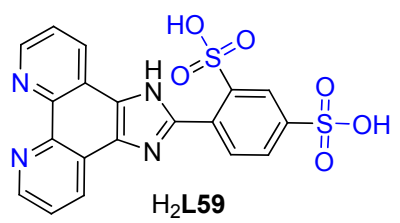

相对于 $\mathrm{Sm}^{3+}$ 而言, $\mathrm{Dy}^{3+}$ 具有更高的最低发射能级 $\left({ }^{4} \mathrm{~F}_{9 / 2}, 21500 \mathrm{~cm}^{-1}\right)$, 因此基于 $\mathrm{Dy}^{3+}$ 的单/同核稀土配合 物/聚合物白光材料甚是少见. 苏成勇等 ${ }^{[2]}$ 通过配体分 子 “推-拉” 电子结构的设计, 实现了配体分子内电荷 转移激发态以及 $\mathrm{T}_{1}$ 激发态协同敏化 $\mathrm{Dy}^{3+}$ 发光, 从而调 制得到光色可调的白光. 组成为 $\left[\mathrm{Dy}(\mathbf{L 6 0})\left(\mathrm{NO}_{3}\right)_{3}\right] \cdot 4 \mathrm{H}_{2} \mathrm{O}$ 的三维 LnOF 材料在激发波长为 265 或 $290 \mathrm{~nm}$ 时, 发射 黄光; 激发波长为 $373 \mathrm{~nm}$ 时发射蓝光; 激发波长为 365 $\mathrm{nm}$ 时得到 CIE 坐标为 $(0.33,0.35)$ 的白光. 而组成为 $\left[\operatorname{Pr}(\mathrm{L61})_{2}\left(\mathrm{NO}_{3}\right)_{3}\right] \cdot \mathrm{CH}_{3} \mathrm{COCH}_{3} \cdot \mathrm{H}_{2} \mathrm{O}$ 的 $\mathrm{LnOF}$ 材料 ${ }^{\left[{ }^{[6]}\right]}$ 在 335 $\mathrm{nm}$ 激发下白光 $\mathrm{CIE}$ 坐标为 $(0.36,0.35)$. 配体 $\mathbf{L 6 2}$ 的同核 聚合物(L62-Ln) ${ }^{[38]}$ 表现出二维网状或一维环链结合的 结构. L62-Pr 和 L62-Dy 均有光色可调的发光行为, 前者 在 $345 \mathrm{~nm}$ 激发下得到 CIE 坐标、 CCT 以及 CRI 分别为 $(0.34,0.30) 、 5468 \mathrm{~K}$ 以及 98 的白光; 后者在 $339 \mathrm{~nm}$ 激 发下相应白光指标分别为 $(0.32,0.33) 、 5461 \mathrm{~K}$ 以及 97.
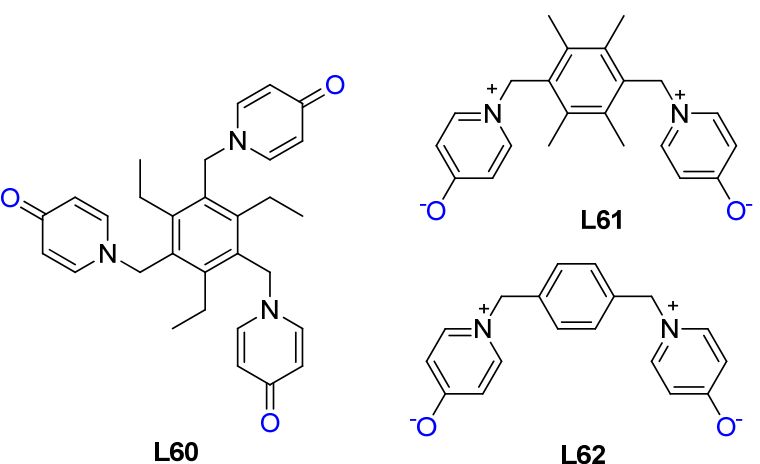

鉴于篇幅问题，其它稀土白光材料的组成以及相关 参数见表 1 .

\section{6 基于稀土配合物/聚合物的其它类型的稀土 白光材料}

冯嘉春等 ${ }^{[109]}$ 将表面乙烯化的碳点 $(\mathrm{CDs})$ 经由自由 基聚合嵌入到 PMMA 链上作为蓝光光源, 稀土配合物 L79 Ln $\left(\mathrm{Ln}=\mathrm{Eu}^{3+}\right.$ 和 $\left.\mathrm{Tb}^{3+}\right)$ 分别与 PMMA 链上羰基氧原 子进一步配位得到聚合物白光薄膜材料 L79-LnPMMA-CDs(图 9). 当嵌入的 CDs、 L79Tb 以及 $\mathbf{L} 79 \mathrm{Eu}$ 质量分数分别为 $0.2 \%, 0.25 \%$ 和 $0.00375 \%$ 时, $400 \mathrm{~nm}$ 激 发下得到 CIE 坐标为 $(0.31,0.32)$ 的白光, 量子产率为 $16.6 \%$.

Holten-Andersen 等 ${ }^{[110]}$ 在 $N, N$-亚甲基双丙烯酸胺交 联剂(MBAA)的存在下, 通过甲基丙烯酸功能化的 CDs 与丙烯酰胺(AA)和丙烯酸(AAc)之间的自由基聚合制备 得到超分子水凝胶 $\mathrm{AA} / \mathrm{AAc}-\mathrm{CDs}$. 在此水凝胶中依次引 入 $\mathrm{Eu}^{3+}$ 和 $\mathrm{Tb}^{3+}$ 以及敏化配体三联吡啶后制备得到发光 凝胶 AA/AAc-CDs-Ln(图 10). 精确控制丙烯酸和功能 化的 $\mathrm{CDs}$ 的质量比为 $3: 1 、 \mathrm{Eu}^{3+} / \mathrm{Tb}^{3+}$ 物质的量之比分 别为 $1: 1,3: 2$ 以及 $2: 1,330 \mathrm{~nm}$ 激发下得到 CIE 坐标 分别为 $(0.27,0.35),(0.29,0.34)$ 以及 $(0.32,0.33)$ 的白光. 研究表明, 这种白光凝胶具有 $\mathrm{pH}$ 值、蒸汽(氯化氢、丙 酮以及氨气)、过渡金属离子以及温度等多刺激响应性. 类似地, Song 等 ${ }^{[111]}$ 以 $\mathrm{Na}_{4}$ EDTA 为碳源, 水热法合成了 表面羰基、羟基和氨基功能化的 CDs, 当在其中掺杂 $\mathrm{Eu}^{3+}$ 和 $\mathrm{Tb}^{3+}$ 时可调制白光. 
表 1 稀土白光材料及其对应地配体、 $\operatorname{Ln}^{3+}$ 的组成、激发波长 $\left(\lambda_{\mathrm{ex}}\right) 、 \mathrm{CIE}$ 坐标、CCT、CRI 以及量子产率 $(\Phi)^{a}$

Table 1 Summary of the ligands, $\mathrm{Ln}^{3+}$ composition, excitation wavelength $\left(\lambda_{\mathrm{ex}} / \mathrm{nm}\right)$, CIE coordinates, correlated color temperature $(\mathrm{CCT})$, color rendering index (CRI) and quantum yields $(\Phi)$ for the white-light-emitting lanthanide-based materials

\begin{tabular}{|c|c|c|c|c|c|c|c|}
\hline Entry & 配体 & $\mathrm{Ln}^{3+}$ 的组成 & $\lambda_{\mathrm{ex}} / \mathrm{nm}$ & $\operatorname{CIE}(x, y)$ & $\mathrm{CCT} / \mathrm{K}$ & CRI & $\Phi / \%$ \\
\hline $1^{[97]}$ & $\mathrm{H}_{3} \mathbf{L 2 8}$ & $\left(\mathrm{Bi}_{0.9}\right) \mathrm{Eu}_{0.005} \mathrm{~Tb}_{0.095}$ & 335 & $(0.33,0.32)$ & - & - & - \\
\hline $2^{[93]}$ & $\mathrm{H}_{2} \mathbf{L 5 7}$ & $\mathrm{Eu}_{0.719} \mathrm{Dy}_{0.281}$ & 372 & $(0.33,0.33)$ & 5505 & - & 1.76 \\
\hline $3^{[93]}$ & $\mathrm{H}_{2} \mathbf{L 5 7}$ & $\mathrm{Eu}_{0.103} \mathrm{~Tb}_{0.871} \mathrm{Gd}_{0.026}$ & 383 & $(0.33,0.34)$ & 5475 & - & 7.93 \\
\hline $4^{[98]}$ & L62 & $\mathrm{Gd}_{0.85} \mathrm{Eu}_{0.06} \mathrm{~Tb}_{0.09}$ & 365 & $(0.35,0.35)$ & 4833 & 73 & - \\
\hline $5^{[98]}$ & L63 & $\mathrm{La}_{0.8} \mathrm{Eu}_{0.1} \mathrm{~Tb}_{0.1}$ & 360 & $(0.35,0.34)$ & 4737 & 97 & - \\
\hline $6^{[98]}$ & L63 & $\mathrm{La}_{0.6} \mathrm{Dy}_{0.2} \mathrm{Sm}_{0.2}$ & 335 & $(0.33,0.32)$ & 5226 & 97 & - \\
\hline $7^{[29]}$ & $\mathrm{H}_{3} \mathbf{L} \mathbf{6 4}$ & $\mathrm{La}_{0.44} \mathrm{Eu}_{0.10} \mathrm{~Tb}_{0.46}$ & 345 & $(0.33,0.33)$ & - & - & 11.22 \\
\hline $8^{[29]}$ & $\mathrm{H}_{3} \mathbf{L} 64$ & $\mathrm{La}_{0.66} \mathrm{Eu}_{0.11} \mathrm{~Tb}_{0.23}$ & 345 & $(0.32,0.31)$ & - & - & 2.85 \\
\hline $9^{[28]}$ & $\mathrm{H}_{4} \mathbf{L} 65$ & $\mathrm{Eu}_{0.006} \mathrm{~Tb}_{0.009} \mathrm{Gd}_{0.985}$ & 380 & $(0.32,0.33)$ & - & - & - \\
\hline $10^{[99]}$ & L16, $\mathrm{H}_{4} \mathbf{L} 66$ & $\mathrm{Eu}_{0.0167} \mathrm{~Tb}_{0.9833}$ & 360 & $(0.32,0.34)$ & - & - & - \\
\hline $11^{[34]}$ & $\mathrm{H}_{4} \mathbf{L} 67$ & $\mathrm{Eu}_{0.015} \mathrm{~Tb}_{0.035} \mathrm{Gd}_{0.95}$ & 340 & $(0.33,0.34)$ & - & - & - \\
\hline $12^{[20]}$ & $\mathrm{H}_{4} \mathbf{L 6 8}$ & $\mathrm{Eu}_{0.10} \mathrm{Gd}_{0.90}$ & 350 & $(0.39,0.38)$ & 3872 & - & 7.6 \\
\hline $13^{[100]}$ & HL69 & $\mathrm{Eu}_{0.52} \mathrm{~Tb}_{0.392} \mathrm{Gd}_{0.088}$ & 375 & $(0.32,0.34)$ & - & - & - \\
\hline $14^{[101]}$ & $\mathrm{H}_{2} \mathbf{L 7 0}$ & $\mathrm{Eu}_{0.0748} \mathrm{~Tb}_{0.9252}$ & 380 & $(0.33,0.33)$ & - & - & - \\
\hline $15^{[102]}$ & $\mathrm{H}_{3} \mathbf{L} 71$ & $\mathrm{Eu}_{0.04} \mathrm{~Tb}_{0.04} \mathrm{Gd}_{0.92}$ & 312 & $(0.33,0.34)$ & - & - & - \\
\hline $16^{[103]}$ & $\mathrm{H}_{3} \mathbf{L} 72$ & $\mathrm{Eu}_{0.1388} \mathrm{~Tb}_{0.2504} \mathrm{Gd}_{0.6108}$ & 355 & $(0.32,0.33)$ & 6261 & 82 & 4.2 \\
\hline $17^{[104]}$ & $\mathrm{H}_{3} \mathbf{L} 73$ & $\mathrm{La}_{0.73} \mathrm{Eu}_{0.24} \mathrm{~Tb}_{0.03}$ & 350 & $(0.33,0.33)$ & - & - & - \\
\hline $18^{[105]}$ & $\mathrm{H}_{4} \mathrm{~L} 74$ & $\mathrm{Sm}_{0.1} \mathrm{~Tb}_{0.04} \mathrm{Dy}_{0.06}$ & 389 & $(0.33,0.35)$ & 4444 & 86.7 & 19 \\
\hline $19^{[106]}$ & $\mathrm{H}_{3} \mathbf{L 7 5}$ & $\left(\mathrm{Pb}_{2}\right) \mathrm{Eu}$ & 400 & $(0.35,0.34)$ & - & - & - \\
\hline $20^{[107]}$ & $\mathrm{H}_{2} \mathrm{L76}$ & $\mathrm{Sm}_{0.2} \mathrm{Eu}_{0.2} \mathrm{~Tb}_{0.6}$ & 322 & $(0.33,0.33)$ & - & - & - \\
\hline $21^{[108]}$ & $\mathrm{H}_{2} \mathbf{L} 77, \mathrm{H}_{2} \mathbf{L} 78$ & $\mathrm{Eu}_{0.1} \mathrm{~Tb}_{0.9}$ & 304 & $(0.30,0.34)$ & - & - & - \\
\hline
\end{tabular}

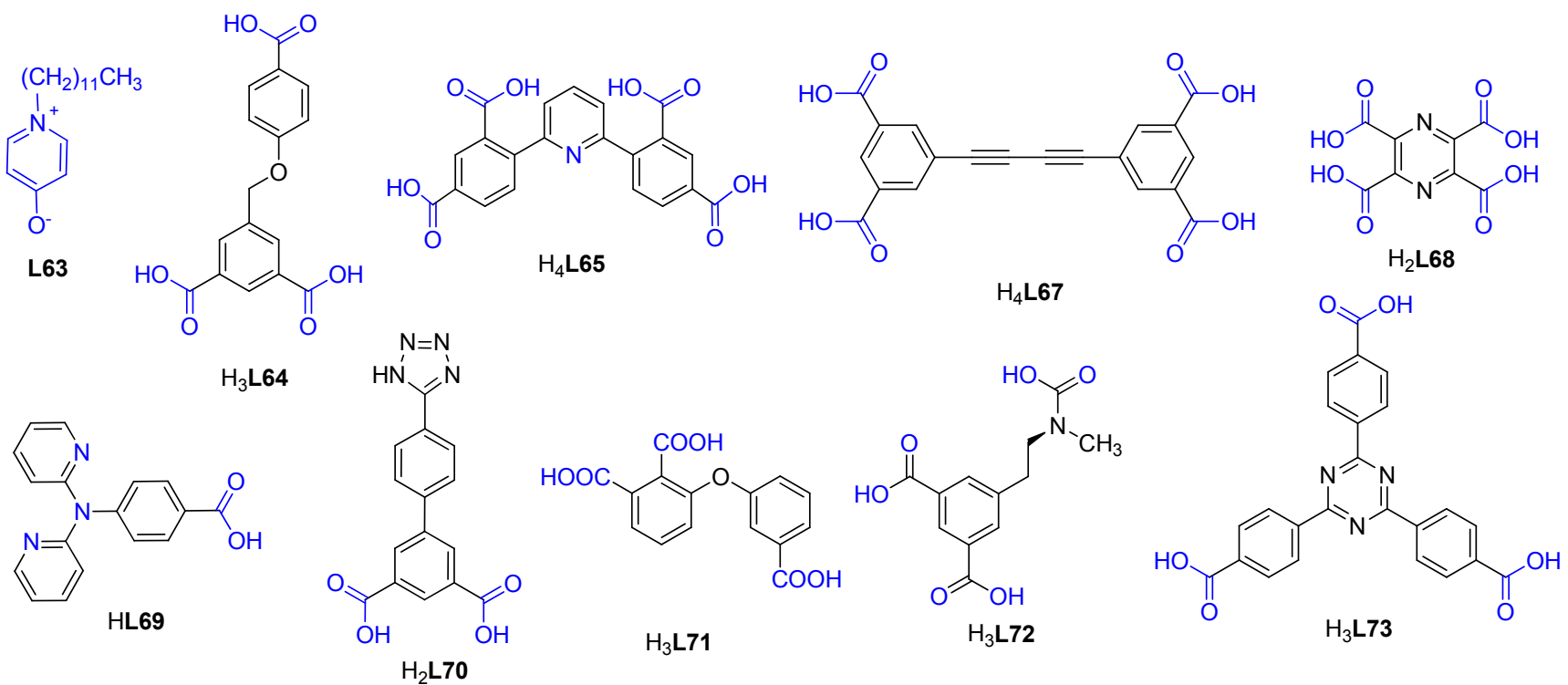<smiles>CC(C)(C)Cc1ccc(C(=O)O)cc1</smiles><smiles>O=C(O)c1ccc(Cn2c(CN(Cc3nc4ccccc4n3Cc3ccc(C(=O)O)cc3)Cc3nc4ccccc4n3Cc3ccc(C(=O)O)cc3)nc3ccccc32)cc1</smiles><smiles>O=C(OC(C(=O)O)C(OC(=O)c1ccccc1)C(=O)O)c1ccccc1</smiles><smiles>O=C(O)c1ccc(C(=O)O)cc1</smiles>

$\mathrm{H}_{2} \mathrm{~L} 77$<smiles></smiles>

$\mathrm{H}_{2} \mathrm{L78}$ 


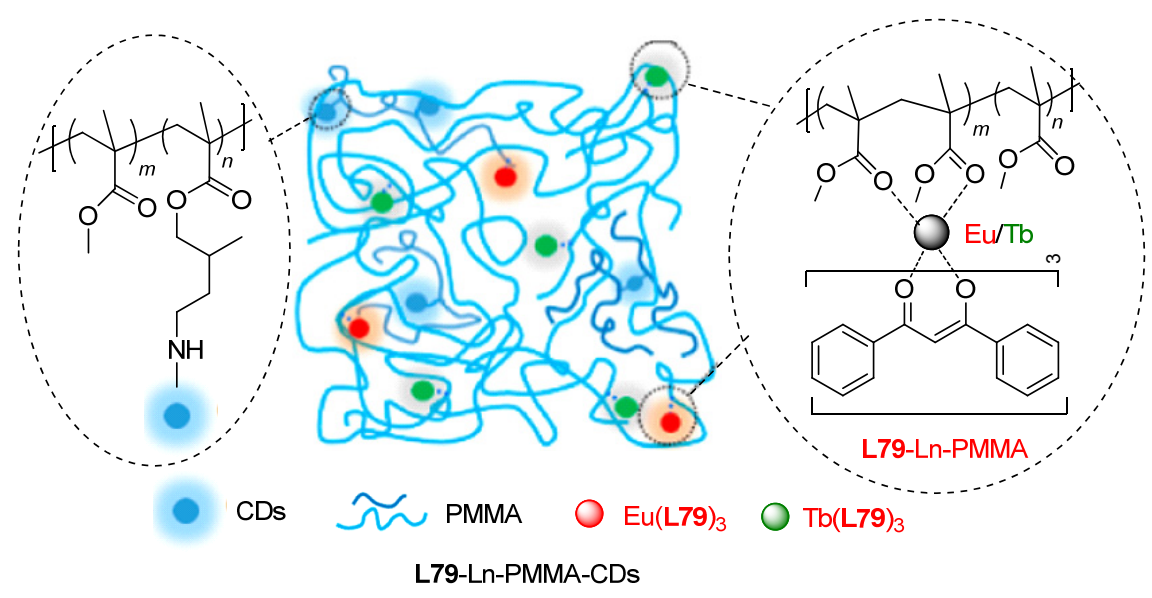

图 9 聚合物 L79-Ln-PMMA-CDs 的组装模式

Figure 9 Assembly model of L79-Ln-PMMA-CDs polymer

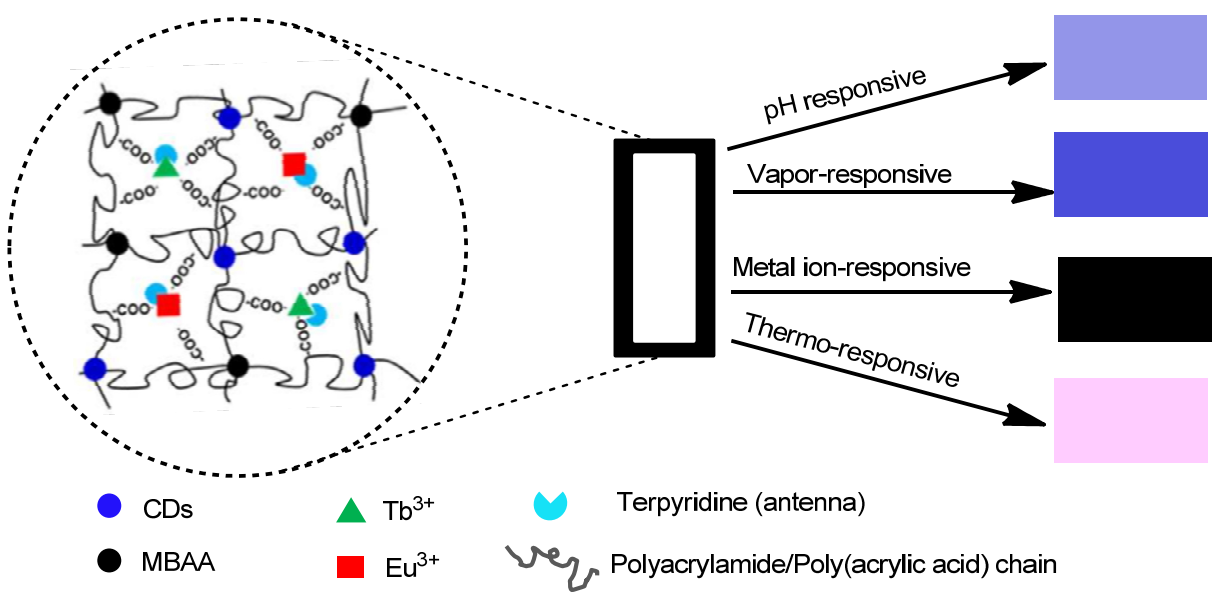

图 10 白光水凝胶 AA/AAc-CDs-Ln 的组装模式及多刺激响应性

Figure 10 Assembly model of white luminescence hydrogel of AA/AAc-CDs-Ln and its multistimuli-responsibilites

\section{7 结论及展望}

近年来，通过科研工作者的不解努力，稀土配合物/ 聚合物白光材料得到了长足发展. 通过对不同类型稀土 白光材料的分类综述, 旨在重点介绍基于 “蓝光和黄光 二基色” 和 “蓝光、绿光和红光三基色” 的白光调制原 理、以及对应的稀土白光材料的制备方法. 分类对比结 果表明, $\mathrm{Eu}^{3+} / \mathrm{Tb}^{3+} / \mathrm{La}^{3+}\left(\mathrm{Gd}^{3+}\right)$ 共掺杂形成的异多核配合 物/聚合物白光材料由于光色平衡而具有较好的发光效 率以及显色性能. 正因如此, 异多核的 LnOFs 材料将在 固态发光、全色显示以及 WLED 器件等领域具有更为 广阔的应用前景. 但遗憾的是, 多种 $\mathrm{Ln}^{3+}$ 共掺杂的调制 策略导致白光的调制成本较高. 相应地, 经由单/同核稀 土配合物/聚合物调制白光时只需一种 $\mathrm{Ln}^{3+}$ 参与, 但其 发光效率和显色性能普遍较差, 也就是说, 在降低白光 调制成本的同时却牺牲了光学性能. 因此, 较高的调制 成本以及较差的光学性能是目前稀土配合物/聚合物白 光材料面临的两大瓶颈问题, 这已成为制约此类材料真
正走向应用的科学难题.

从根本上解决目前稀土白光材料存在的两大瓶颈 问题，关键在于配体分子的结构调控. 在配体分子的设 计中应该兼顾配体与 $\mathrm{Ln}^{3+}$ 间 “光色互补以及能量匹配原 则” . 首先, 配体分子与 $\mathrm{Ln}^{3+}$ 适当的能极差既要保证有 效的能量传递，同时还要部分保留基于配体中心的蓝光 发射. 其次, 通过芳香 $\pi$-体系 “推-拉” 电子结构、激基 缔合物等配体结构的设计，突破传统的从配体三重激发 态能量转移敏化路径, 实现配体多种激发态(如 S1, ILCT, MLCT 等)对 $\mathrm{Ln}^{3+}$ 协同敏化发光. 再次, 通过配体 分子中多重组装位点、辅助配体的引入以及凝胶化等策 略调控组装体组装模式，避免因高能键的振动而造成的 非辐射衰减. 另外, 借助于组装体特殊的构筑方式如外 延生长法、有机配体与高分子链共聚的方法以及 $\mathrm{LnOFs}$ 的独特结构改善白光材料的光学性能以及光学品质. 因 此，如何调制光学性能以及显色性能优异的白光，将是 单/同核稀土配合物/聚合物白光材料研究面临的挑战和 机遇，发展并调控不同激发态能量转移敏化路径势必会 
成为稀土白光材料新的研究方向.

\section{References}

[1] Cui, Y.; Chen, B.; Qian, G. Coord. Chem. Rev. 2014, 273, 76.

[2] Pan, M.; Liao, W.-M.; Yin, S.-Y.; Sun, S.-S.; Su, C.-Y. Chem. Rev. 2018, $118,8899$.

[3] Wang, M.-S.; Guo, G.-C. Chem. Commun. 2016, 52, 13194

[4] Wang, Z.; Wang, Z.; Lin, B.; Hu, X.; Wei, Y.; Zhang, C.; An, B.; Wang, C.; Lin, W. ACS Appl. Mater. Interfaces 2017, 9, 35253.

[5] SeethaLekshmi, S.; Ramya, A. R.; Reddy, M. L. P.; Varughese, S. J. Photochem. Photobiol. C 2017, 33, 109.

[6] Shang, M.; Li, C.; Lin, J. Chem. Soc. Rev. 2014, 43, 1372.

[7] Yin, W.; Bai, X.; Chen, P.; Zhang, X.; Su, L.; Ji, C.; Gao, H.; Song, H.; Yu, W. W. ACS Appl. Mater. Interfaces 2018, 10, 43824.

[8] Kang, X.; Song, S.; Wang, H.; Ling, D.; Lü, W. ACS Omega 2018, 3,16714 .

[9] He, Z.; Zhao, W.; Lam, J. W. Y.; Peng, Q.; Ma, H.; Liang, G.; Shuai, Z.; Tang, B. Z. Nat. Commun. 2017, 8, 416.

[10] Sun, C.-Y.; Wang, X.-L.; Zhang, X.; Qin, C.; Li, P.; Su, Z.-M.; Zhu, D.-X.; Shan, G.-G.; Shao, K.-Z.; Wu, H.; Li, J. Nat. Commun. 2013, 4, 3717.

[11] Liu, Y.; Pan, M.; Yang, Q.-Y.; Fu, L.; Li, K.; Wei, S.-C.; Su, C.-Y. Chem. Mater. 2012, 24, 1954.

[12] Singh, K.; Boddula, R.; Vaidyanathan, S. Inorg. Chem. 2017, 56, 9376.

[13] Wu, J.; Zhang, H.; Du, S. J. Mater. Chem. C 2016, 4, 3364.

[14] Zhang, Z.; He, Y.-N.; Liu, L.; Lü, X.-Q.; Zhu, X.-J.; Wong, W.-K.; Pan, M.; Su, C.-Y. Chem. Commun. 2016, 52, 3713.

[15] Zheng, X.-L.; Liu, Y.; Pan, M.; Lü, X.-Q.; Zhang, J.-Y.; Zhao, C.-Y.; Tong, Y.-X.; Su, C.-Y. Angew. Chem., Int. Ed. 2007, 46, 7399 .

[16] Weissman, S. I. J. Chem. Phys. 1942, 10, 214.

[17] Steemers, F. J.; Verboom, W.; Reinhoudt, D. N.; Vander Tol, E. B.; Verhoeven, J. W. J. Am. Chem. Soc. 1995, 117, 9408.

[18] Latva, M.; Takalo, H.; Mukkala, V. M.; Matachescu, C.; Rodriguez-Ubis, J. C.; Kankare, J. J. Lumin. 1997, 75, 149.

[19] Li, R.; Wang, T. Chin. J. Lumin. 2018, 39, 425 (in Chinese). (李焕荣，王天任，发光学报，2018，39, 425.)

[20] Zhang, F.; Yan, P.; Li, H.; Zou, X.; Hou, G.; Li, G. Dalton Trans. 2014, 43, 12574.

[21] Barry, D. E.; Caffrey, D. F.; Gunnlaugsso, T. Chem. Soc. Rev. 2016, $45,3244$.

[22] Zhang, J.; Li, H.; Chen, P.; Sun, W.; Gao, T.; Yan, P. J. Mater. Chem. C 2015, 3, 1799

[23] Bünzli, J.-C. G. Coord. Chem. Rev. 2015, 293 294, 19.

[24] Yang, Q.-Y.; Wu, K.; Jiang, J.-J.; Hsu, C.-W.; Pan, M.; Lehn, J.-M.; $\mathrm{Su}, \mathrm{C} . \mathrm{Y}$. Chem. Commun. 2014, 50, 7702.

[25] Li, S.; Guo, N.; Liang, Q.-M.; Deng, H.-X. Chin. J. Inorg. Chem. 2017, 33, 543 (in Chinese). (李硕, 郭宁, 梁启蒙, 邓虹霄，无机化学学报，2017, 33, 543.)

[26] Cui, Y.; Yue, Y.; Qian, G.; Chen, B. Chem. Rev. 2012, 112, 1126.

[27] Zhao, Y.-W.; Zhang, F.-Q.; Zhang, X.-M. ACS Appl. Mater. Interfaces 2016, 8, 24123.

[28] Yang, Y.; Chen, L.; Jiang, F.; Yu, M.; Wan, X.; Zhang, B.; Hong, M. J. Mater. Chem. C 2017, 5, 1981.

[29] An, Y.-Y.; Lu, L.-P.; Feng, S.-S.; Zhu, M.-L. CrystEngComm 2018 , 20, 2043.

[30] Huang, J.-J.; Yu, J.-H.; Bai, F.-Q.; Xu, J.-Q. Cryst. Growth Des. 2018, 18, 5353 .

[31] Li, X.; Wang, Y.; Xie, J.; Yin, X.; Silver, M. A.; Cai, Y.; Zhang, H.; Chen, L.; Bian, G.; Diwu, J.; Chai, Z.; Wang, S. Inorg. Chem. 2018, 57,8714 .

[32] Zhang, Y.-H.; Li, X.; Song, S.; Yang, H.-Y.; Ma, D.; Liu, Y.-H. CrystEngComm 2014, 16, 8390.

[33] Cha, Y.-E.; Li, X.; Ma, D.; Huo, R. Eur. J. Inorg. Chem. 2014, 2969.

[34] Rong, J.; Zhang, W.; Bai, J. RSC Adv. 2016, 6, 103714.
[35] Coppo, P.; Duati, M.; Kozhevnikov, V. N.; Hofstraat, J. W.; Cola, L. De. Angew. Chem., Int. Ed. 2005, 44, 1806.

[36] Leng, J.; Li, H.; Chen, P.; Sun, W.; Gao, T.; Yan, P. Dalton Trans. 2014, 43, 12228.

[37] Al-Rasbi, N. K.; Adams, H.; Suliman, F. O. Dyes Pigm. 2014, 104, 83.

[38] Liao, W.-M.; Li, C.-J.; Wu, X.; Zhang, J.-H.; Wang, Z.; Wang, H.-P.; Fan, Y.-N.; Pan, M.; Su, C.-Y. J. Mater. Chem. C 2018, 6, 3254.

[39] An, R.; Zhao, H.; Hu, H.-M.; Wang, X.; Yang, M.-L.; Xue, G. Inorg. Chem. 2016, 55, 871.

[40] Ma, D.; Li, X.; Huo, R. J. Mater. Chem. C 2014, 2, 9073.

[41] Zhang, H.; Shan, X.; Zhou, L.; Lin, P.; Li, R.; Ma, E.; Guo, X.; Du, S. J. Mater. Chem. C 2013, 1,888

[42] Zhou, Y.; Zhang, H.-Y.; Zhang, Z.-Y.; Liu, Y. J. Am. Chem. Soc. 2017, 139, 7168 .

[43] Robin, A. Y.; Fromm, K. M. Coord. Chem. Rev. 2006, 250, 2172.

[44] Al-Bahri, A.; Al-Zakwani, A. S.; Al-Farsi, S. M.; Al-Rasbi, N. K. ChemistrySelect 2016, 1, 1393.

[45] Liu, C.-L.; Zhang, R.-L.; Lin, C.-S.; Zhou, L.-P.; Cai, L.-X.; Kong, J.-T.; Yang, S.-Q.; Han, K.-L.; Sun, Q.-F. J. Am. Chem. Soc. 2017, $139,12474$.

[46] Xu, H.; Wang, J.; Wei, Y.; Xie, G.; Xue, Q.; Deng, Z.; Huang, W. J. Mater. Chem. C 2015, 3, 1893 .

[47] Yang, H.; Zhang, Y.; Li, Y.; Wang, J.; Li, X.; Song, J.; Zhang, B.; Feng, Y. Chin. J. Org. Chem. 2017, 37, 1991 (in Chinese). (杨贺玮, 张宇哲, 李艳杰, 王京翔, 李小萌, 宋健, 张宝, 冯亚 青, 有机化学, 2017, 37, 1991.)

[48] Chen, W.; Fan, R.; Zhang, H.; Dong, Y.; Wang, P.; Yang, Y. Dalton Trans. 2017, 46, 4265 .

[49] Kotova, O.; Comby, S.; Lincheneau, C.; Gunnlaugsson, T. Chem. Sci. 2017, 8, 3419 .

[50] Gu, Z.-G.; Chen, Z.; Fu, W.-Q.; Wang, F.; Zhang, J. ACS Appl. Mater. Interfaces 2015, 7, 28585.

[51] Feng, W.-X.; Yin, S.-Y.; Pan, M.; Wang, H.-P.; Fan, Y.-N.; Lü, X.-Q.; Su, C.-Y. J. Mater. Chem. C 2017, 5, 1742.

[52] Chen, P.; Holten-Andersen, N. Adv. Opt. Mater. 2015, 3, 1041.

[53] Wang, S.; Wu, T.-Z.; Park, H.-J.; Peng, T.; Cao, L.-X.; Mellerup, S. K.; Yang, G.-Q.; Wang, N.; Peng, J.-B. Adv. Opt. Mater. 2016, 4, 1882 .

[54] Boddula, R.; Singh, K.; Giri, S.; Vaidyanathan, S. Inorg. Chem. 2017, 56, 10127

[55] Liu, L.; Fu, G.; Li, B.; Lü, X.; Wong, W.-K.; Jonesc, R. A. RSC Adv. 2017, 7, 6762 .

[56] Zhang, A.; Sun, N.; Li, L.; Yang, Y.; Zhao, X.; Jia, H.; Liu, X.; Xu, B. J. Mater. Chem. C 2015, 3, 9933.

[57] Tigaa, R. A.; Aerken, X.; Fuchs, A.; de Bettencourt-Dias, A. Eur. J. Inorg. Chem. 2017, 5310.

[58] Liu, H.; Chu, T.; Rao, Z.; Wang, S.; Yang, Y.; Wong, W.-T. Adv. Opt. Mater. 2015, 3, 1545.

[59] Wang, H.-Y.; Ding, Z.-J.; Li, Z.; Zhang, Y.; Li, H. Colloid Polym. Sci. 2018, 296, 53 .

[60] Feng, C.; Sun, J.-W.; Yan, P.-F.; Li, Y.-X.; Liu, T.-Q.; Sun, Q.-Y.; Li, G.-M. Dalton Trans. 2015, 44, 4640

[61] Xiao, Y.; Wang, S.-H.; Zheng, F.-K.; Wu, M.-F.; Xu, J.; Liu, Z.-F.; Chen, J.; Lia, R.; Guo, G.-C. CrystEngComm 2016, 18, 721.

[62] Guo, P.-H.; Meng, Y.; Chen, Y.-C.; Li, Q.-W.; Wang, B.-Y.; Leng, J.-D.; Bao, D.-H.; Jia, J.-H.; Tong, M.-L.; J. Mater. Chem. C 2014, 2,8858

[63] Sutar, P.; Maji, T. K. Inorg. Chem. 2017, 56, 9417.

[64] Kumar, P.; Soumya, S.; Prasad, E. ACS Appl. Mater. Interfaces 2016, 8,8068 .

[65] Silva, J. Y. R.; da Luz, L. L.; Mauricio, F. G. M.; Vasconcelos Alves, I. B.; Ferro, J. N. de S.; Barreto, E.; Júnior, S. A. ACS Appl. Mater. Interfaces 2017, 9, 16458.

[66] Mahapatra, T. S.; Singh, H.; Maity, A.; Dey, A.; Pramanik, S. K.; Suresh, E.; Das, A. J. Mater. Chem. C 2018, 6, 9756.

[67] Gao, X.; Chang, S.; Liu, H.; Liu, Z. Eur. J. Inorg. Chem. 2016 
2837.

[68] Gai, Y.; Guo, Q.; Xiong, K.; Jiang, F.; Li, C.; Li, X.; Chen, Y.; Zhu, C.; Huang, Q.; Yao, R.; Hong, M. Cryst. Growth Des. 2017, 17, 940.

[69] Fan, L.; Fan, W.; Li, B.; Zhao, X.; Zhang, X. New J. Chem. 2016, 40, 10440.

[70] Huang, W.; Pan, F.; Liu, Y.; Huang, S.; Li, Y.; Yong, J.; Li, Y.; Kirillov, A. M.; Wu, D. Inorg. Chem. 2017, 56, 6362.

[71] Ma, M.-L.; Qin, J.-H.; Ji, C.; Xu, H.; Wang, R.; Li, B.-J.; Zang, S.-Q.; Hou, H.-W.; Batten, S. R. J. Mater. Chem. 2014, 2, 1085.

[72] Mondal, S. S.; Behrens, K.; Matthes, P. R.; Schönfeld, F.; Nitsch, J.; Steffen, A.; Primus, P.-A.; Kumke, M. U.; Müller-Buschbaum, K.; Holdt, H.-J. J. Mater. Chem. C 2015, 3, 4623.

[73] Xie, W.; Zhang, S.-R.; Du, D.-Y.; Qin, J.-S.; Bao, S.-J.; Li, J.; Su, Z.-M.; He, W.-W.; Fu, Q.; Lan, Y.-Q. Inorg. Chem. 2015, 54, 3290.

[74] He, H.; Sun, F.; Borjigin, T.; Zhao, N.; Zhu, G. Dalton Trans. 2014, $43,3716$.

[75] Du, X.; Fan, R.; Qiang, L.; Wang, P.; Song, Y.; Xing, K.; Zheng, X.; Yang, Y. Cryst. Growth Des. 2017, 17, 2746.

[76] Zhou, Z.; Li, Q.; Han, Y.; Xing, X.; Du, S. RSC Adv. 2015, 5, 97831.

[77] Li, X.-Y.; Shi, W.-J.; Wang, X.-Q.; Ma, L.-N.; Hou, L.; Wang, Y.-Y. Cryst. Growth Des. 2017, 17, 4217.

[78] Song, S.; Li, X.; Zhang, Y.-H.; Huo, R.; Ma, D. Dalton Trans. 2014, 43, 5974.

[79] Huo, R.; Li, X.; Ma, D. CrystEngComm 2015, 17, 3838.

[80] Ma, D.; Huo, R.; Li, X. CrystEngComm 2015, 17, 6575.

[81] da Luz, L. L.; F. B.; Viana, L.; Oliveira da Silva, G. C.; Gatto, C. C.; Fontes, A. M.; Malta, M.; Weber, I. T.; Rodrigues, M. O.; Júnior, S. A. CrystEngComm 2014, 16, 6914.

[82] Zhu, M.; Hao, Z.-M.; Song, X.-Z.; Meng, X.; Zhao, S.-N.; Song, S.-Y.; Zhang, H.-J. Chem. Commun. 2014, 50, 1912.

[83] Jia, J.; Xu, J.; Wang, S.; Wang, P.; Gao, L.; Yu, M.; Fan, Y.; Wang, L. CrystEngComm 2015, 17, 6030.

[84] Zhao, D.; Rao, X.; Yu, J.; Cui, Y.; Yang, Y.; Qian, G. J. Solid State Chem. 2015, 230, 287.

[85] Shen, L.; Yang, L.; Fan, Y.; Wang, L.; Xu, J. CrystEngComm 2015, $17,9363$.

[86] Zhang, H.; Shan, X.; Ma, Z.; Zhou, L.; Zhang, M.; Lin, P.; Hu, S.; Ma, E.; Li R.; Du, S. J. Mater. Chem. C 2014, 2, 1367.
[87] Tang, Q.; Liu, S.; Liu, Y.; He, D.; Miao, J.; Wang, X.; Ji, Y.; Zheng, Z. Inorg. Chem. 2014, 53, 289.

[88] Zhou, Y.; Yan, B. Inorg. Chem. 2014, 53, 3456.

[89] Zhou, Y.; Yan, B. Nanoscale 2015, 7, 4063.

[90] Lu, Y.; Yan, B. Chem. Commun. 2014, 50, 15443.

[91] Wang, Y.; Xing, S.-H.; Bai, F.-Y.; Xing, Y.-H.; Sun, L.-X. Inorg. Chem. 2018, 57, 12850.

[92] Wei, Y.; Li, Q.; Sa, R.; Wu, K. Chem. Commun. 2014, 50, 1820.

[93] Han, Y.; Yan, P.; Sun, J.; An, G.; Yao, X.; Li, Y.; Li, G. Dalton Trans. 2017, 46, 4642.

[94] Hao, J.; Yan, B. J. Mater. Chem. C 2014, 2, 6758.

[95] Wei, Y.; Sa, R.; Wu, K. Dalton Trans. 2016, 45, 18661.

[96] Du, B.-B.; Zhu, Y.-X.; Pan, M.; Yue, M.-Q.; Hou, Y.-J.; Wu, K.; Zhang, L.-Y.; Chen, L.; Yin, S.-Y.; Fan, Y.-N.; Su, C.-Y. Chem. Commun. 2015, 51, 12533.

[97] Xu, L.; Xu, Y.; Li, X.; Wang, Z.; Sun, T.; Zhang, X. Dalton Trans. 2018, 47, 16696.

[98] Pan, M.; Du, B.-B.; Zhu, Y.-X.; Yue, M.-Q.; Wei, Z.-W.; Su, C.-Y. Chem.-Eur. J. 2016, 22, 2440.

[99] Liu, J.; Sun, W.; Liu, Z. RSC Adv. 2016, 6, 25689.

[100] Ramya, A. R.; Varughese, S.; Reddy, M. L. P. Dalton Trans. 2014, 43, 10940.

[101] Wu, L.-L.; Zhao, J.; Wang, H.; Wang, J. CrystEngComm 2016, 18 , 4268.

[102] Zhang, H.; Fan, R.; Dong, Y.; Chen, W.; Du, X.; Wang, P.; Yang, Y. CrystEngComm 2016, 18, 3711.

[103] Wang, W.; Wang, R.; Ge, Y.; Wu, B. RSC Adv. 2018, 8, 42100

[104] Rong, J.; Zhang, W.; Bai, J. CrystEngComm 2016, 18, 7728.

[105] Li, H.; Liu, H.-B.; Tao, X.-M.; Su, J.; Ning, P.-F.; Xu, X.-F.; Zhou, Y.; Gu, W.; Liu, X. Dalton Trans. 2018, 47, 8427.

[106] Yan, C.; Chen, L.; Pan, M.; Zhang, L.-Y.; Yin, S.-Y.; Zhu, Y.-X.; Wu, K.; Hou, Y.-J.; Su, C.-Y. New J. Chem. 2015, 39, 3770.

[107] Li, Y.; Li, S.; Yan, P.; Wang, X.; Yao, X.; An, G.; Li, G. Chem. Commun. 2017, 53, 5067.

[108] Park, J.; Oh, M. CrystEngComm 2016, 18, 8372.

[109] Chen, B.; Feng, J. J. Phys. Chem. C 2015, 119, 7865.

[110] Zhu, Q.; Zhang, L.; Vliet, K. V.; Miserez, A.; Holten-Andersen, N. ACS Appl. Mater. Interfaces 2018, 10, 10409.

[111] Zhang, T.; Zhai, Y.; Wang, H.; Zhu, J.; Xu, L.; Dong, B.; Song, H. RSC Adv. 2016, 6, 61468. 